\title{
CONVERGENCE OF LOCALLY DIVERGENCE-FREE DISCONTINUOUS-GALERKIN METHODS FOR THE INDUCTION EQUATIONS OF THE 2D-MHD SYSTEM*
}

\author{
Nicolas Besse ${ }^{1}$ AND Dietmar KRÖNER ${ }^{2}$
}

\begin{abstract}
We present the convergence analysis of locally divergence-free discontinuous Galerkin methods for the induction equations which appear in the ideal magnetohydrodynamic system. When we use a second order Runge Kutta time discretization, under the CFL condition $\Delta t \sim h^{4 / 3}$, we obtain error estimates in $L^{2}$ of order $\mathcal{O}\left(\Delta t^{2}+h^{m+1 / 2}\right)$ where $m$ is the degree of the local polynomials.
\end{abstract}

Mathematics Subject Classification. 76W05, 65J10.

Received: January 17, 2005. Revised: July 4, 2005.

\section{INTRODUCTION}

The magnetohydrodynamic (MHD) equations modelize electrically conducting fluid flow in which the electromagnetic forces can be of the same order or even greater than hydrodynamic ones. The ideal MHD system which combines the equations of gas dynamics with Maxwell equations in which relativistic, viscous and resistive effects are neglected can be written in the following three dimensional conservative form

$$
\begin{array}{rlll}
\partial_{t} \rho+\nabla \cdot(\rho \mathbf{u}) & =0 & \text { (mass conservation) } \\
\partial_{t}(\rho \mathbf{u})+\nabla \cdot\left(\rho \mathbf{u} \otimes \mathbf{u}+\left(p+\frac{1}{2}|\mathbf{B}|^{2}\right) \mathcal{I}-\mathbf{B} \otimes \mathbf{B}\right) & =0 & \text { (momentum conservation) } \\
\partial_{t} \mathbf{B}+\nabla \cdot(\mathbf{u} \otimes \mathbf{B}-\mathbf{B} \otimes \mathbf{u}) & =0 & \text { (induction equation) } \\
\partial_{t}(\rho e)+\nabla \cdot\left(\left(\rho e+p+\frac{1}{2}|\mathbf{B}|^{2}\right) \mathbf{u}-\mathbf{B}(\mathbf{u} \cdot \mathbf{B})\right) & =0 & \text { (energy conservation) } \\
\nabla \cdot \mathbf{B} & =0 & \text { (divergence constraint) }
\end{array}
$$

where $\rho$ is the density, $\mathbf{u}$ the velocity field, $\mathbf{B}$ the magnetic field, $p$ the pressure, $e$ the total energy and $\mathcal{I}$ identity matrix. If the initial data are divergence free, that is to say that $\nabla \cdot \mathbf{B}_{0}=0$, then an exact solution will satisfy this constraint for all the times. For smooth solution this is obvious because the induction equation can be

\footnotetext{
Keywords and phrases. Magnetohydrodynamics, discontinuous-Galerkin methods, convergence analysis.

* The authors acknowledge financial support from the HYKE project No HPRN-CT-2002-00282 on "Hyperbolic and Kinetic Equations: Asymptotics, Numerics, Applications", funded by European Union.

1 Institut de Recherche Mathematique Avancée, Université Louis Pasteur, CNRS, 7 rue René Descartes, 67084 Strasbourg Cedex, France. besse@math.u-strasbg.fr

2 Institut für Angewandte Mathematik Universität Freiburg, Hermann-Herder-Str. 10, 79104 Freiburg i. Br., Germany. dietmar@mathematik.uni-freiburg.de
} 
rewritten as $\partial_{t} \mathbf{B}+\operatorname{curl}(\mathbf{B} \times \mathbf{u})=0$ and we have the identity $\nabla \cdot(\nabla \times \cdot)=0$. The numerical preservation of the $\nabla \cdot \mathbf{B}=0$ condition is an important and much debated problem for numerical MHD codes [3, 4,10-12, 20, 22]. Because $\nabla \cdot \mathbf{B}$ errors arise in numerical simulations and may increase in time, numerical instabilities can appear and lead to unphysical behaviour of the system. For example numerically incorrect magnetic field topologies lead to unphysical plasma transport orthogonal to the magnetic field. The non enforcement of the $\nabla \cdot \mathbf{B}$ constraint leads to the loss of momentum and energy conservation and allows fictitious forces to develop parallel to the magnetic field. These effects are discussed in $[3,4]$.

In this paper we present the convergence analysis and error estimates of locally divergence-free Runge Kutta discontinuous Galerkin schemes for smooth solutions of the two dimensional induction equation which arises in the MHD system

$$
\frac{\partial \mathbf{B}}{\partial t}+\operatorname{curl}(\mathbf{B} \times \mathbf{u})=0
$$

where $\mathbf{B}=\left(B_{x}(t, \mathbf{x}), B_{y}(t, \mathbf{x})\right)$ is the magnetic field, and $\mathbf{u}=\left(u_{x}(t, \mathbf{x}), u_{y}(t, \mathbf{x})\right)$ is the velocity field, with the notations $\mathbf{x}=(x, y)$. We assume for this paper that the velocity field $\mathbf{u}$ is given.

The construction and the convergence analysis of this discontinuous Galerkin method rest on three ingredients. First we rewrite the induction equation as a Friedrichs system. Then we write a discontinuous Galerkin formulation of this new system in which we choose upwind flux as definition of the flux at the cell interface. In order to have a locally divergence free scheme we use piecewise solenoidal functions that are totally discontinuous across interface cells but which are pointwise divergence free on each element. This basis functions were developed in $[2,16]$ in the context of nonconforming finite element method for the stationary Navier-Stokes equations and were also used in [17]. We also use the Nedelec finite element in $\mathrm{H}$ (curl) [19] in two dimensions which is obtained by rotating the Raviart-Thomas element [21] by $\pi / 2$. Then we used the framework developed in [6,24] to show the convergence and obtained error estimates for our scheme. In [7] the authors develop a locally divergence free discontinuous Galerkin scheme for numerically solving the Maxwell equation. They also show an error estimate of the form $\mathcal{O}\left(h^{k+1 / 2}\right)$ where $k$ is the degree of the local polynomials for a scheme semi-discretized in space only.

\section{THE NUMERICAL SCHEME}

\subsection{The Friedrichs formulation}

Thanks to the divergence constraint, $\nabla \cdot \mathbf{B}=0$, the induction equation (1) can be rewritten as the following Friedrichs system:

where

$$
\frac{\partial \mathbf{B}}{\partial t}+\frac{\partial\left(\mathcal{A}_{x} \mathbf{B}\right)}{\partial x}+\frac{\partial\left(\mathcal{A}_{y} \mathbf{B}\right)}{\partial y}+\mathcal{C} \mathbf{B}=0 \quad \text { in } \Omega \times[0, T]
$$

and

$$
\mathcal{A}_{x}(t, \mathbf{x})=\left(\begin{array}{cc}
u_{x}(t, \mathbf{x}) & 0 \\
0 & u_{x}(t, \mathbf{x})
\end{array}\right), \quad \mathcal{A}_{y}(t, \mathbf{x})=\left(\begin{array}{cc}
u_{y}(t, \mathbf{x}) & 0 \\
0 & u_{y}(t, \mathbf{x})
\end{array}\right),
$$

$$
\mathcal{C}(t, \mathbf{x})=-\left(\begin{array}{ll}
\partial_{x} u_{x}(t, \mathbf{x}) & \partial_{y} u_{x}(t, \mathbf{x}) \\
\partial_{x} u_{y}(t, \mathbf{x}) & \partial_{y} u_{y}(t, \mathbf{x})
\end{array}\right) .
$$

Sometimes we will use the notation $\left(\boldsymbol{v}_{1}, \boldsymbol{v}_{2}\right)$ instead of $\left(\boldsymbol{v}_{x}, \boldsymbol{v}_{y}\right)$ where $\boldsymbol{v}$ is a vector or matrix fields. For suitable boundary conditions on $\partial \Omega$ Friedrichs [14] has proved the existence and uniqueness in $L^{2}(\Omega)$ of a weak and strong solution to (2), under appropriate smoothness conditions and the additional assumption

$$
\mathcal{C}+\mathcal{C}^{T}+\frac{\partial \mathcal{A}_{x}}{\partial x}+\frac{\partial \mathcal{A}_{y}}{\partial y} \geq \alpha \mathcal{I}, \quad \text { in } \Omega,
$$

with $\alpha$ a strictly positive constant. We suppose that $\mathbf{u} \in L^{\infty}\left(0, T ; \mathscr{C}^{\infty}(\Omega)\right), \nabla \cdot \mathbf{u} \in L^{\infty}\left(0, T ; L^{\infty}(\Omega)\right)$ and $\mathcal{C} \in L^{\infty}\left(0, T ; \mathscr{C}^{\infty}(\Omega)\right)$. We recall that if $\mathbf{B}_{0} \in \mathbf{H}^{s}\left(\mathbb{R}^{2}\right)$ with $s \in \mathbb{R}$, then the system (2) admits a unique weak solution $\mathbf{B} \in \mathscr{C}^{0}\left(\left[0,+\infty\left[; \mathbf{H}^{s}\left(\mathbb{R}^{2}\right)\right) \cap \mathscr{C}^{1}\left(\left[0,+\infty\left[; \mathbf{H}^{s-1}\left(\mathbb{R}^{2}\right)\right)\right.\right.\right.\right.$ (see [1]). In particular, if $s>2$ the weak solution lies in $\mathscr{C}^{1}\left(\left[0,+\infty\left[\times \mathbb{R}^{2}\right)\right.\right.$ and is in fact a classical solution. 
Remark 1. The condition (3) can be easily satisfied by using $\widetilde{\mathbf{B}}(t, \mathbf{x})=e^{-\lambda t} \mathbf{B}(t, \mathbf{x})$, with $\lambda>0$ instead of $\mathbf{B}$. Then $\widetilde{\mathbf{B}}$ satisfies

$$
\frac{\partial \widetilde{\mathbf{B}}}{\partial t}+\frac{\partial\left(\mathcal{A}_{x} \widetilde{\mathbf{B}}\right)}{\partial x}+\frac{\partial\left(\mathcal{A}_{y} \widetilde{\mathbf{B}}\right)}{\partial y}+\widetilde{\mathcal{C}} \widetilde{\mathbf{B}}=0 \quad \text { in } \Omega \times[0, T]
$$

where $\widetilde{\mathcal{C}}=\mathcal{C}+\lambda \mathcal{I}$. Since we assume $\nabla \mathbf{u} \in L^{\infty}(\Omega)$ we can choose $\lambda$ sufficiently large such that condition (3) is satisfied for (4).

\subsection{The approximation spaces}

First we introduce the space $\ell^{\infty}(0, T ; X)$ defined by

$$
\ell^{\infty}(0, T ; X):=\left\{f:\left\{t^{0}, \ldots, t^{N_{T}}\right\} \rightarrow X \mid\|f\|_{\ell^{\infty}(0, T ; X)}=\max _{1 \leq n \leq N_{T}}\left\|f\left(t^{n}\right)\right\|_{X}<\infty\right\}
$$

where $X$ denotes a functional space. For a subdomain $D \subset \Omega, H^{m}(D)$ denotes the usual Sobolev space which is a Hilbert space with the inner product $(u, v)_{m, D}=\sum_{|\alpha| \leq m} \int_{D} \partial^{\alpha} u \partial^{\alpha} v \mathrm{dx}, \quad u, v \in H^{m}(D)$ and the square of seminorm $|v|_{H^{m}(D)}^{2}=\sum_{|\alpha|=m} \int_{D}\left|\partial^{\alpha} v\right|^{2}$. Then we define the space $\mathbf{H}^{m}(D)=\left(H^{m}(D)\right)^{2}$ with inner product $(\mathbf{v}, \mathbf{w})_{m, D}=\sum_{i=1}^{2}\left(v_{i}, w_{i}\right)_{m, D}$, and the solenoidal vector fields $\mathbf{S}^{m}(D)=\left\{\mathbf{v} \in \mathbf{H}^{m}(D) \mid \nabla \cdot \mathbf{v}=0\right.$ in $\left.D\right\}$, $m \geq 1$. Let $\mathcal{T}_{h}$ a family of partitions of $\Omega$ that possesses properties described in $[2,16]$. For integer $k \geq 0$, $\mathbb{P}^{k}(D)$ will denote the linear space of polynomials in two variables, of degree less than or equal to $k$ on $D$. Then we define $\mathbb{P}^{k}(D)=\left(\mathbb{P}^{k}(D)\right)^{2}$ and $V^{k}(D)=\left\{\mathbf{v} \in \mathbb{P}^{k}(D) \mid \nabla \cdot \mathbf{v}=0\right.$ in $\left.D\right\}$. We have $V^{k}(D) \subset \mathbf{S}^{m}(D)$ for all $m \geq 1$. For $k \geq 0$ we define $V_{h}^{k}(\Omega)=\prod_{K \in \mathcal{T}_{h}} V^{k}(K)$ where $K$ is a finite element (or cell) and $N$ the number of finite elements. The way of constructing local bases for $V^{k}$ and hence for $V_{h}^{k}$ are described in [2, 16]. For example, for $k=1$ the bases functions can be constructed using the set of functions

$$
\Xi^{1}=\left\{\left(\begin{array}{l}
1 \\
0
\end{array}\right),\left(\begin{array}{l}
0 \\
1
\end{array}\right),\left(\begin{array}{l}
y \\
0
\end{array}\right),\left(\begin{array}{l}
0 \\
x
\end{array}\right),\left(\begin{array}{l}
x \\
-y
\end{array}\right)\right\} .
$$

To obtain a basis function for $k=2$ it suffices to augment the above set by

$$
\Xi^{2}=\left\{\left(\begin{array}{l}
y^{2} \\
0
\end{array}\right),\left(\begin{array}{l}
0 \\
x^{2}
\end{array}\right),\left(\begin{array}{l}
x^{2} \\
-2 x y
\end{array}\right),\left(\begin{array}{l}
-2 x y \\
y^{2}
\end{array}\right)\right\}
$$

Then we see that the basis functions for the approximation space $V_{h}^{k}$ can be constructed by adding suitable terms to $V_{h}^{k-1}$.

Remark 2. In the case where $\mathcal{T}_{h}$ is a triangulation in $\mathbb{R}^{2}$ the spaces $V^{k}(K)$ are equivalent to the $\operatorname{spaces} R T_{k}^{0}(K)$ defined by $R T_{k}^{0}(K)=\left\{\mathbf{v} \in R T_{k}(K) \mid \operatorname{div} \mathbf{v}=0\right\}$, where $R T_{k}(K)$ is the usual Raviart-Thomas space on $K$ used to approximate $\mathbf{H}(\operatorname{div})$. In fact each element of $V^{k}(K)$ or $R T_{k}^{0}(K)$ is constructed as the curl of a stream function which belongs to $\mathbb{P}^{k}(K)$ and the dimension of the both space is equal to $\operatorname{dim}\left(\mathbb{P}^{k}(K)-1\right)=(k+1)(k+4) / 2$.

The spaces $V_{h}^{m}$ possess optimal approximation properties in relation to the spaces $\mathbf{S}^{m}(\Omega)$ which has been proved in [2]. We recall some of those.

Theorem 1. Let $m \geq 0$. If $\mathbf{v} \in \mathbf{H}^{m+1}(K)$, then there exists $\boldsymbol{\chi} \in \mathbb{P}^{m}(K)$ such that

$$
\|\mathbf{v}-\chi\|_{\mathbf{H}^{j}(K)} \leq C h_{K}^{m+1-j}|\mathbf{v}|_{\mathbf{H}^{m+1}(K)}, \quad 0 \leq j \leq m+1
$$

where $h_{K}$ is the diameter of the largest ball contained in the cell $K$.

Let $m \geq 0$. If $\mathbf{v} \in \mathbf{S}^{m+1}(K)$, then there exists $\chi \in V^{m}(K)$ such that

$$
\|\mathbf{v}-\chi\|_{\mathbf{H}^{j}(K)} \leq C h_{K}^{m+1-j}|\mathbf{v}|_{\mathbf{H}^{m+1}(K)}, \quad 0 \leq j \leq m+1 .
$$


Proof. See [2].

Now we define the local $\mathbf{L}^{2}$ projection operator $\pi_{h}$ from $\mathbf{S}^{m}(\Omega)$ onto $V_{h}^{k}(\Omega)$, with $m \geq 1$ by the equations $\pi_{h}=\sum_{K \in \mathcal{T}_{h}} \pi_{K} \mathbb{1}_{K}(\mathbf{x})$ and

$$
\int_{K}\left(\pi_{K} \mathbf{v}-\mathbf{v}\right) \cdot \phi=0, \quad \forall \phi \in V^{k}(K), \forall K \in \mathcal{T}_{h} .
$$

where $\mathbb{1}_{K}(\mathbf{x})$ denotes the characteristic functions of $K$ which is equal to 1 on $K$ and 0 elsewhere. Thanks to the properties of the partition $\mathcal{T}_{h}$ (see [2]) there exists a constant $\sigma$ such that $h_{K} \leq \sigma h, \forall K \in \mathcal{T}_{h}$. For example we can take $h=\min _{K \in \mathcal{T}_{h}} h_{K}$. Then we have the following approximation result.

Theorem 2. Let $\mathcal{T}_{h}$ be a partition of $\Omega$ satisfying the properties assumed in [2], i.e.

i) $\cup_{K \in \mathcal{T}_{h}} \bar{K}=\bar{\Omega}, K \cap Q=\emptyset, K, Q \in \mathcal{T}_{h}, K \neq Q$.

ii) $\exists \mu_{1}$ a constant independent of $N$ and $K \in \mathcal{T}_{h}$ such that $\rho_{K} \leq \mu_{1} h_{K}$ where $\rho_{K}$ is the diameter of the smallest ball containing $K$.

iii) $\exists \mu_{2}$ a constant independent of $N$ and $K \in \mathcal{T}_{h}$ such that $h_{Q} \leq \mu_{2} \rho_{K}$ whenever $Q$ and $K$ have a common 1-dimensional interface.

iv) $K$ is a regular domain in $\mathbb{R}^{2}$, i.e. the divergence theorem holds on $K$.

If $\mathbf{v} \in \mathbf{S}^{m+1}(\Omega)$ then there exists a constant $C$ such that

$$
\left\|\pi_{h} \mathbf{v}-\mathbf{v}\right\|_{\mathbf{L}^{2}(\Omega)} \leq C h^{m+1}|\mathbf{v}|_{\mathbf{H}^{m+1}(\Omega)} .
$$

Moreover we have the following inverse inequalities

$$
\left|\mathbf{v}_{h}\right|_{\mathbf{H}^{1}(K)} \leq \frac{C}{h}\left\|\mathbf{v}_{h}\right\|_{\mathbf{L}^{2}(K)}, \quad\left|\mathbf{v}_{h}\right|_{\mathbf{L}^{2}(\partial K)} \leq \frac{C}{h^{1 / 2}}\left\|_{\mathbf{v}_{h}}\right\|_{\mathbf{L}^{2}(K)}, \quad \forall \mathbf{v}_{h} \in V^{m}(K)
$$

and the following approximation result

$$
\left\|\pi_{h} \mathbf{v}-\mathbf{v}\right\|_{\mathbf{H}^{s}(K)} \leq C h^{m+1-s}|\mathbf{v}|_{\mathbf{H}^{m+1}(K)}, \quad \forall \mathbf{v} \in \mathbf{S}^{m+1}(\Omega) \quad s \in \mathbb{R}^{+}, \quad s \leq m+1
$$

Proof. See Appendix 5.

Now we introduce another discontinuous interpolation operator $\Pi_{h}$ based on the two-dimensional Nédélec element in $\mathrm{H}$ (curl) (see [19]). Let $\widetilde{\mathbb{P}}_{k}$ denote the space of homogeneous polynomials of degree $k$ in $\mathbb{R}^{2}$ and consider the following subspace of $\mathbb{P}^{k}, \mathcal{R}_{k}=\mathbb{P}^{k-1} \oplus S_{k}$ where $S_{k}$ is defined by $S_{k}=\left\{\mathbf{p} \in \widetilde{\mathbb{P}}_{k} ; \mathbf{p}(\mathbf{x}) \cdot \mathbf{x}=0\right.$, for all $\left.\mathbf{x}=\left(x_{1}, x_{2}\right)\right\}$. Then we define $W^{k}(K)=\left\{\mathbf{v} \in \mathcal{R}_{k}(K)\right\}$ and $W_{h}^{k}(\Omega)=\prod_{K \in \mathcal{T}_{h}} W^{k}(K)$. Following [15], if we define the two sets of moments of $\mathbf{v} \in \mathbf{H}^{s}$ with $s \geq 1 / 2$ on $K: \mathcal{M}_{e}(\mathbf{v})=\left\{\int_{e}\left(\mathbf{v} \times \boldsymbol{\nu}_{e}\right) q \mathrm{~d} \Gamma \quad \forall q \in \mathbb{P}^{k-1}(e)\right.$ for all edges $e$ of $K\}$ where $\boldsymbol{\nu}_{e}$ is the outward unit normal to the edges $e$ of $K$, and $\mathcal{M}_{K}(\mathbf{v})=\left\{\int_{K} \mathbf{v} \cdot \mathbf{q d} \mathbf{x}, \quad \forall \mathbf{q} \in \mathbb{P}^{k-2}(K)\right\}$, then a vector field $\mathbf{v}$ of $\mathcal{R}_{k}$ is entirely determined in a triangle $K$ by its two sets of moments: $\mathcal{M}_{e}(\mathbf{v}), \mathcal{M}_{K}(\mathbf{v})$. Moreover the tangential components of $\mathbf{v}$ on a given edge $e$ of $K$ depend only the moments $\mathcal{M}_{e}(\mathbf{v})$ defined on that edges. Besides we have the following approximation properties (see $[15,19])$ :

Lemma 1. Let $\mathbf{v} \in \mathbf{H}^{m}($ curl; $\Omega)$, then there exists a constant $C$ independent of $h$ such that

$$
\begin{gathered}
\left\|\mathbf{v}-\Pi_{h} \mathbf{v}\right\|_{\mathbf{L}^{2}(K)} \leq C h^{m}|\mathbf{v}|_{\mathbf{H}^{m}(K)} \\
\left\|\mathbf{v}-\Pi_{h} \mathbf{v}\right\|_{\mathbf{H}(\text { curl } ; K)} \leq C h^{m}\left(|\mathbf{v}|_{\mathbf{H}^{m}(K)}+|\operatorname{curl} \mathbf{v}|_{\mathbf{H}^{m}(K)}\right)
\end{gathered}
$$

and

$$
\left\|\left(\mathbf{v}-\Pi_{h} \mathbf{v}\right) \times \boldsymbol{\nu}_{e}\right\|_{H^{s}(e)} \leq C h^{m-s}|\mathbf{v}|_{\mathbf{H}^{m}(e)}, \quad \forall e \in \partial K \quad s \leq m
$$

with $\Pi_{h} \mathbf{v}_{\left.\right|_{K}}=\Pi_{K} \mathbf{v}$ where $\Pi_{K} \mathbf{v} \in W^{m}(K)$ is determined by $\mathcal{M}_{K}\left(\Pi_{K} \mathbf{v}-\mathbf{v}\right)=\{0\}$, and $\mathcal{M}_{e}\left(\Pi_{K} \mathbf{v}-\mathbf{v}\right)=\{0\}$. 
If $m=1$ then $\mathcal{R}_{1}=\mathbb{P}^{0} \oplus S_{1}$ where $S_{1}=\operatorname{span}\left(x^{\perp}\right)$ and then it is obvious that div $\mathcal{R}_{1} \equiv 0$. Then the vector fields of $\mathcal{R}_{1}$ is divergence free. Unfortunately the spaces $\mathcal{R}_{k}$ with $k>1$ are not divergence free spaces. Now we present a trace result and a continuous embedding which will be useful later. Let $K$ a convex polygon with Lipschitz boundary in $\mathbb{R}^{2}$. First we define the space $\mathcal{V}(K)$ and $\mathcal{W}(K)$ by $\mathcal{W}(K)=\{\mathbf{v} \in \mathbf{H}(\operatorname{curl} ; K) \cap$ $\left.\mathbf{H}(\operatorname{div} ; K), \mathbf{v} \times \boldsymbol{\nu} \in L^{2}(\partial K)\right\}$ equipped with the norm $\|\mathbf{v}\|_{\mathcal{W}(K)}=\|\mathbf{v}\|_{L^{2}(K)}+\|\operatorname{curl} \mathbf{v}\|_{L^{2}(K)}+\|\operatorname{div} \mathbf{v}\|_{L^{2}(K)}+$ $\|\mathbf{v} \times \boldsymbol{\nu}\|_{L^{2}(\partial K)}$ and $\mathcal{V}(K)=\left\{\mathbf{v} \in \mathbf{H}(\operatorname{curl} ; K) \cap \mathbf{H}(\operatorname{div} ; K), \quad \mathbf{v} \cdot \boldsymbol{\nu} \in L^{2}(\partial K)\right\}$ equipped with the norm $\|\mathbf{v}\|_{\mathcal{V}(K)}=\|\mathbf{v}\|_{L^{2}(K)}+\|\operatorname{curl} \mathbf{v}\|_{L^{2}(K)}+\|\operatorname{div} \mathbf{v}\|_{L^{2}(K)}+\|\mathbf{v} \cdot \boldsymbol{\nu}\|_{L^{2}(\partial K)}$. It is proved in [8,9] that $\mathcal{V}(K)=\mathcal{W}(K)$ and then that the mapping $\mathbf{v} \longrightarrow \gamma_{0} \mathbf{v}$ defined on $\mathscr{D}(\bar{K})$ has a unique linear continuous extension as an operator from $\mathcal{W}(K)$ onto $\mathbf{L}^{2}(\partial K)$, where $\gamma_{0} \mathbf{v}$ is the boundary values of $\mathbf{v}$ on $\partial K$. Now let us define $\mathcal{Q}(K)=\left\{\mathbf{v} \in \mathbf{H}(\operatorname{curl} ; K) \cap \mathbf{H}(\operatorname{div} ; K), \quad \mathbf{v} \times \boldsymbol{\nu}_{e} \in H^{1 / 2}(e) \forall e \in \partial K\right\} \quad$ equipped with the norm $\|\mathbf{v}\|_{\mathcal{Q}(K)}=\|\mathbf{v}\|_{L^{2}(K)}+\|\operatorname{curl} \mathbf{v}\|_{L^{2}(K)}+\|\operatorname{div} \mathbf{v}\|_{L^{2}(K)}+\sum_{e \in \partial K}\left\|\mathbf{v} \times \boldsymbol{\nu}_{e}\right\|_{H^{1 / 2}(e)}$. Then we have the following theorem where a proof can be found in [13]

Theorem 3. Let $K$ be a convex polygon in $\mathbb{R}^{2}$ with Lipschitz boundary. Then we have the continuous embedding:

$$
\mathcal{Q}(K) \hookrightarrow \mathbf{H}^{1}(K)
$$

\subsection{The discontinuous Galerkin method}

In this section we describe our discontinuous Galerkin method. If we take the scalar product of the equation (1) with a test function $\varphi$, integrate the scalar product on a cell $K$ and use the following Green formula

$$
\int_{K} v \partial_{i} w \mathrm{~d} \mathbf{x}=-\int_{K} w \partial_{i} v \mathrm{~d} \mathbf{x}+\sum_{e \in \partial K} \int_{e} w v \nu_{e, K}^{i} \mathrm{~d} \Gamma
$$

where $\boldsymbol{\nu}_{e, K}=\left(\nu_{e, K}^{1}, \nu_{e, K}^{2}\right)^{T}=\left(\nu_{e, K}^{x}, \nu_{e, K}^{y}\right)^{T}$ denotes the outward unit normal to the face $e$ of $K$, we obtain

$$
\partial_{t}\left(\int_{K} \mathbf{B} \cdot \boldsymbol{\varphi} \mathrm{d} \mathbf{x}\right)-\sum_{i=1}^{2} \int_{K} \mathcal{A}_{i} \mathbf{B} \cdot \partial_{i} \boldsymbol{\varphi} \mathrm{d} \mathbf{x}+\sum_{i=1}^{2} \sum_{e \in \partial K} \int_{e} \mathcal{A}_{i} \mathbf{B} \cdot \varphi \nu_{e, K}^{i} \mathrm{~d} \Gamma+\int_{K} \mathcal{C} \mathbf{B} \cdot \boldsymbol{\varphi} \mathrm{d} \mathbf{x}=0
$$

Now we replace respectively $\mathbf{B}$ and $\boldsymbol{\varphi}$ by $\mathbf{B}_{h}$ and $\boldsymbol{\varphi}_{h}$ in $(15)$ where $\mathbf{B}_{h}, \boldsymbol{\varphi}_{h} \in V_{h}^{k}(\Omega)$. Nevertheless the terms arising from the boundary of the cell $K$ in (15) are not well defined or have no sense since $\mathbf{B}_{h}$ and $\boldsymbol{\varphi}_{h}$ are discontinuous on the boundary $\partial K$ of the element $K$. Then we replace these terms by a numerical upwind flux that we are going to define in the following lines. Let us define $\mathcal{A}_{e, K}(t, \mathbf{x})=\sum_{i=1}^{2}\left(\mathcal{A}_{i}\right)_{\left.\right|_{e}} \nu_{e, K}^{i}$ and $C_{e, K}(t, \mathbf{x})=-\left(\mathcal{A}_{e, K}(t, \mathbf{x})\right)^{-}, D_{e, K}(t, \mathbf{x})=\left(\mathcal{A}_{e, K}(t, \mathbf{x})\right)^{+}$where $A^{-}$and $A^{+}$denote respectively the negative and the positive part of $A$. Then we define the upwind numerical flux $g\left(\nu_{e, K}, \mathbf{v}, \mathbf{w}\right)=-C_{e, K} \mathbf{w}+D_{e, K} \mathbf{v}$. By noting that $|A|=A^{+}-A^{-}$and $A=A^{+}+A^{-}$we can rewrite $g\left(\nu_{e, K}, \mathbf{v}, \mathbf{w}\right)$ as the Lax-Friedrichs flux $g\left(\nu_{e, K}, \mathbf{v}, \mathbf{w}\right)=\mathcal{A}_{e, K} \frac{(\mathbf{v}+\mathbf{w})}{2}-\left|\mathcal{A}_{e, K}\right| \frac{(\mathbf{w}-\mathbf{v})}{2}$, where $\mathbf{v}$ (resp. w) denotes the interior (resp. exterior) value of the solution from the cell $K$. Now we obtain the following semi-discretized scheme in space

$$
\partial_{t}\left(\int_{K} \mathbf{B}_{h} \cdot \boldsymbol{\varphi}_{h} \mathrm{~d} \mathbf{x}\right)-\sum_{i=1}^{2} \int_{K} \mathcal{A}_{i} \mathbf{B}_{h} \cdot \partial_{i} \boldsymbol{\varphi}_{h} \mathrm{~d} \mathbf{x}+\sum_{i=1}^{2} \sum_{e \in \partial K} \int_{e} g\left(\boldsymbol{\nu}_{e, K}, \mathbf{B}_{K}, \mathbf{B}_{K_{e}}\right) \cdot \boldsymbol{\varphi}_{K} \mathrm{~d} \Gamma+\int_{K} \mathcal{C} \mathbf{B}_{h} \cdot \boldsymbol{\varphi}_{h} \mathrm{~d} \mathbf{x}=0
$$

for all $\varphi_{h} \in V_{h}^{k}$ where $K_{e}$ is the neighbourhood of $K$ along the face $e$. Let $T$ be the final time and $\Delta t=T / N_{T}$ the time step. Now we use a second order Runge-Kutta scheme for the discretization in time. Then the fully 
discretized scheme reads

$$
\begin{aligned}
\int_{K} \mathbf{Y}_{h}^{n} \cdot \varphi_{h} \mathrm{~d} \mathbf{x} & =\int_{K} \mathbf{B}_{h}^{n} \cdot \varphi_{h} \mathrm{~d} \mathbf{x}+\Delta t \mathcal{F}_{K}^{n}\left(\mathbf{B}_{h}^{n}, \varphi_{h}\right) \\
\int_{K} \mathbf{B}_{h}^{n+1} \cdot \varphi_{h} \mathrm{~d} \mathbf{x} & =\int_{K}\left(\frac{1}{2} \mathbf{B}_{h}^{n}+\frac{1}{2} \mathbf{Y}_{h}^{n}\right) \cdot \boldsymbol{\varphi}_{h} \mathrm{~d} \mathbf{x}+\frac{\Delta t}{2} \mathcal{F}_{K}^{n+1}\left(\mathbf{Y}_{h}^{n}, \varphi_{h}\right)
\end{aligned}
$$

where

$$
\mathcal{F}_{K}^{n}\left(\mathbf{W}, \boldsymbol{\varphi}_{h}\right)=\sum_{i=1}^{2} \int_{K} \mathcal{A}_{i}^{n} \mathbf{W}^{n} \cdot \partial_{i} \boldsymbol{\varphi}_{h} \mathrm{~d} \mathbf{x}+\sum_{i=1}^{2} \sum_{e \in \partial K} \int_{e} g^{n}(\mathbf{W})_{e} \cdot \boldsymbol{\varphi}_{K} \mathrm{~d} \Gamma-\int_{K} \mathcal{C}^{n} \mathbf{W}^{n} \cdot \boldsymbol{\varphi}_{h} \mathrm{~d} \mathbf{x}
$$

with

$$
g^{n}(\mathbf{W})_{e}=g^{n}\left(\nu_{e, K}, \mathbf{W}_{K}^{n}, \mathbf{W}_{K_{e}}^{n}\right)=\mathcal{A}_{e, K}^{n} \frac{\left(\mathbf{W}_{K}^{n}+\mathbf{W}_{K_{e}}^{n}\right)}{2}-\left|\mathcal{A}_{e, K}^{n}\right| \frac{\left(\mathbf{W}_{K_{e}}^{n}-\mathbf{W}_{K}^{n}\right)}{2}
$$

and $\mathcal{A}_{e, K}^{n}=\mathcal{A}_{e, K}\left(t^{n}, \mathbf{x}\right), \mathcal{C}^{n}=\mathcal{C}\left(t^{n}, \mathbf{x}\right)$. Let $\mathcal{X}^{k}$ be $V^{k}$ or $W^{k}$. If we expand $\mathbf{B}_{h}$ and $\boldsymbol{\varphi}_{h}$, that is to say

$$
\mathbf{B}_{h}^{n}(\mathbf{x})=\sum_{K \in \mathcal{T}_{h}} \mathbf{B}_{K}^{n}(\mathbf{x}) \mathbb{1}_{K}(\mathbf{x}), \quad \mathbf{B}_{K}^{n}(\mathbf{x})=\sum_{i=1}^{\operatorname{dim}\left(\mathcal{X}^{k}\right)} \sigma_{K}^{i, n} \Psi_{i}(\mathbf{x}), \quad \mathbf{Y}_{K}^{n}(\mathbf{x})=\sum_{i=1}^{\operatorname{dim}\left(\mathcal{X}^{k}\right)} \theta_{K}^{i, n} \Psi_{i}(\mathbf{x})
$$

with $\Psi_{i} \in \mathcal{X}^{k}$, then we obtain the scheme

$$
\begin{cases}{\left[\Theta_{K}\right]^{n}} & =\left[\Sigma_{K}\right]^{n}+\Delta t \mathcal{L}_{K}^{n}\left(\left[\Sigma_{h}\right]^{n}\right) \\ {\left[\Sigma_{K}\right]^{n+1}} & =\frac{1}{2}\left(\left[\Sigma_{K}\right]^{n}+\left[\Theta_{K}\right]^{n}\right)+\Delta t \mathcal{L}_{K}^{n+1}\left(\left[\Theta_{h}\right]^{n}\right)\end{cases}
$$

where $\left[\Sigma_{K}\right]^{n}=\left(\sigma_{K}^{1, n}, \ldots, \sigma_{K}^{\operatorname{dim}}\left(\mathcal{X}^{k}\right), n\right)^{T},\left[\Sigma_{h}\right]^{n}=\left(\sigma^{1, n}, \ldots, \sigma^{\operatorname{card}\left(\mathcal{T}_{h}\right) \operatorname{dim}\left(\mathcal{X}^{k}\right), n}\right)^{T}$ and for all $i \in\left\{1, \ldots, \operatorname{dim}\left(\mathcal{X}^{k}\right)\right\}$

$$
\begin{aligned}
{\left[\mathcal{L}_{K}^{n}\left(\left[\Sigma_{h}\right]^{n}\right)\right]_{i}=} & \sum_{l=1}^{2} \sum_{m, j=1}^{\operatorname{dim}\left(\mathcal{X}^{k}\right)} \sigma_{K}^{j, n} \int_{K} \mathcal{A}_{l}^{n}(\mathbf{x}) \Psi_{j}(\mathbf{x}) \cdot\left(\mathcal{M}_{K}^{-T}\right)_{m, i} \partial_{l} \Psi_{m}(\mathbf{x}) \mathrm{d} \mathbf{x} \\
& +\sum_{i=1}^{2} \sum_{e \in \partial K} \int_{e} \sum_{m, j=1}^{\operatorname{dim}\left(\mathcal{X}^{k}\right)}\left[\sigma_{K}^{j, n} D_{e, K}^{n}(\mathbf{x}(\Gamma))-\sigma_{K_{e}}^{j, n} C_{e, K}^{n}(\mathbf{x}(\Gamma))\right] \Psi_{j}(\mathbf{x}(\Gamma)) \cdot\left(\mathcal{M}_{K}^{-T}\right)_{m, i} \Psi_{m}(\mathbf{x}(\Gamma)) \mathrm{d} \Gamma \\
& -\sum_{m, j=1}^{\operatorname{dim}\left(\mathcal{X}^{k}\right)} \sigma_{K}^{j, n} \int_{K} \mathcal{C}^{n}(\mathbf{x}) \Psi_{j}(\mathbf{x}) \cdot\left(\mathcal{M}_{K}^{-T}\right)_{m, i} \Psi_{m}(\mathbf{x}) \mathrm{d} \mathbf{x}
\end{aligned}
$$

with $\left(\mathcal{M}_{K}\right)_{i j}=\int_{K} \Psi_{i}(\mathbf{x}) \cdot \Psi_{j}(\mathbf{x}) \mathrm{d} \mathbf{x}$.

\section{Analysis of the SEMI-DiscRetized SCHEME IN SPACE}

In this section we show the $L^{2}$-stability, the convergence and present some error estimates for the semidiscretized scheme in space, continuous in time.

Theorem 4. Let $\mathbf{u}$ and $\mathbf{B}_{0}$ sufficiently, regular, typically we consider that $\mathbf{u} \in L^{\infty}\left(\left[0,+\infty\left[; W^{1, \infty}\left(\mathbb{R}^{2}\right)\right)\right.\right.$ and $\mathbf{B}_{0} \in \mathbf{H}^{m+1}\left(\mathbb{R}^{2}\right)$ then there exists a constant $C=C\left(\|\nabla \mathbf{u}\|_{L^{\infty}([0, T] \times \Omega)}, T\right)$ independent of $h$ such that

$$
\left\|\mathbf{B}_{h}\right\|_{L^{\infty}\left(0, T ; \mathbf{L}^{2}(\Omega)\right)} \leq C\left\|\mathbf{B}_{h}(0)\right\|_{\mathbf{L}^{2}(\Omega)},
$$


where $\mathbf{B}_{h}(0) \in V_{h}^{m}$ or $\mathbf{B}_{h}(0) \in W_{h}^{m}$ with $m=1$. Let $\left[\mathbf{B}_{h}\right]_{e}$ be the jump of $\mathbf{B}_{h}$ across an edge ef $\mathcal{E}_{h}$, the set of all the edges of the partition $\mathcal{T}_{h}$ then, there exists a constant $C=C\left(\|\nabla \mathbf{u}\|_{L^{\infty}([0, T] \times \Omega)}, T,\|\mathbf{B}\|_{L^{\infty}\left(0, T ; \mathbf{H}^{m+1}\left(\mathbb{R}^{2}\right)\right)}\right)$ independent of $h$ such that

$$
\left\|\mathbf{B}-\mathbf{B}_{h}\right\|_{L^{\infty}\left(0, T ; \mathbf{L}^{2}(\Omega)\right)}+\sqrt{\int_{0}^{T} \sum_{e \in \mathcal{E}_{h}} \int_{e}\left|\mathcal{A}_{e}\right|\left[\mathbf{B}_{h}\right]_{e} \cdot\left[\mathbf{B}_{h}\right]_{e} \mathrm{~d} \Gamma \mathrm{d} t} \leq C h^{m+\eta}
$$

where $\eta=\frac{1}{2}$ if $\mathbf{B}_{h} \in V_{h}^{m}$ and $\eta=-\frac{1}{2}$ if $\mathbf{B}_{h} \in W_{h}^{m}$ with $m=1$.

Proof. The stability result is proved in the Appendix 5. We will show simultaneously the convergence of the scheme and derivate some error estimates. First we begin to derivate estimates which are valid as well for $\mathbf{B}_{h} \in V_{h}^{m}$ than $\mathbf{B}_{h} \in W_{h}^{1}$. Then we continue the proof by distinguishing the case where $\mathbf{B}_{h} \in V_{h}^{m}$ and the case where $\mathbf{B}_{h} \in W_{h}^{1}$. First by noting that

$$
\mathcal{L}_{h}\left(\mathbf{B}_{h}, \boldsymbol{\varphi}_{h}\right)=0 \quad \text { and } \quad \mathcal{L}_{h}\left(\mathbf{B}, \boldsymbol{\varphi}_{h}\right)=0 \quad \forall \boldsymbol{\varphi}_{h} \in V_{h}^{m}(\Omega) \text { or } \forall \boldsymbol{\varphi}_{h} \in W_{h}^{1}(\Omega)
$$

then

$$
\mathcal{L}_{h}\left(\mathbf{e}_{h}, \boldsymbol{\varphi}_{h}\right)=0, \quad \forall \boldsymbol{\varphi}_{h} \in V_{h}^{m}(\Omega) \text { or } \forall \boldsymbol{\varphi}_{h} \in W_{h}^{1}(\Omega)
$$

where $\mathbf{e}_{h}=\mathbf{B}-\mathbf{B}_{h}$. By noting that $\pi_{h} \mathbf{B}_{h}=\mathbf{B}_{h}$ and $\pi_{h} \mathbf{e}_{h}-\mathbf{e}_{h}=\pi_{h}\left(\mathbf{B}-\mathbf{B}_{h}\right)-\left(\mathbf{B}-\mathbf{B}_{h}\right)$, then using (19) we obtain

$$
\mathcal{L}_{h}\left(\pi_{h} \mathbf{e}_{h}, \pi_{h} \mathbf{e}_{h}\right)=\mathcal{L}_{h}\left(\pi_{h} \mathbf{e}_{h}-\mathbf{e}_{h}, \pi_{h} \mathbf{e}_{h}\right)=\mathcal{L}_{h}\left(\pi_{h} \mathbf{B}-\mathbf{B}, \pi_{h} \mathbf{e}_{h}\right)
$$

From the stability analysis done in Appendix 5 the left hand side of (20) is the expression

$$
\begin{aligned}
\mathcal{L}_{h}\left(\pi_{h} \mathbf{e}_{h}, \pi_{h} \mathbf{e}_{h}\right)=\frac{1}{2}\left\|\pi_{h} \mathbf{e}_{h}(T)\right\|_{\mathbf{L}^{2}(\Omega)}^{2}-\frac{1}{2}\left\|\pi_{h} \mathbf{e}_{h}(0)\right\|_{\mathbf{L}^{2}(\Omega)}^{2} & +\frac{1}{2} \int_{0}^{T} \sum_{e \in \mathcal{E}_{h}} \int_{e}\left|\mathcal{A}_{e}\right|\left[\pi_{h} \mathbf{e}_{h}\right]_{e} \cdot\left[\pi_{h} \mathbf{e}_{h}\right]_{e} \mathrm{~d} \Gamma \mathrm{d} t \\
& +\int_{0}^{T} \int_{\Omega} \mathcal{C} \pi_{h} \mathbf{e}_{h} \cdot \pi_{h} \mathbf{e}_{h} \mathrm{~d} \mathbf{x} \mathrm{d} t+\frac{1}{2} \int_{0}^{T} \int_{\Omega} \nabla \cdot \mathbf{u}\left\|\pi_{h} \mathbf{e}_{h}\right\|_{2}^{2} \mathrm{~d} \mathbf{x} \mathrm{d} t .
\end{aligned}
$$

Now it remains to estimate the right hand side of (20) that is to say find an estimate for

$$
\mathcal{L}_{h}\left(\pi_{h} \mathbf{B}-\mathbf{B}, \boldsymbol{\varphi}_{h}\right), \quad \forall \boldsymbol{\varphi}_{h} \in V_{h}^{m}(\Omega) \text { or } \forall \varphi_{h} \in W_{h}^{1}(\Omega) .
$$

Let us decompose $\mathcal{L}_{h}\left(\pi_{h} \mathbf{B}-\mathbf{B}, \varphi_{h}\right)$ in several terms which will be estimated in the sequel.

$$
\begin{aligned}
\mathcal{L}_{h}\left(\pi_{h} \mathbf{B}-\mathbf{B}, \boldsymbol{\varphi}_{h}\right)= & \int_{0}^{T} \int_{\Omega} \partial_{t}\left(\pi_{h} \mathbf{B}-\mathbf{B}\right) \cdot \boldsymbol{\varphi}_{h} \mathrm{~d} \mathbf{x} \mathrm{d} t \\
& -\sum_{i=1}^{2} \int_{0}^{T} \int_{\Omega} \mathcal{A}_{i}\left(\pi_{h} \mathbf{B}-\mathbf{B}\right) \cdot \partial_{i} \boldsymbol{\varphi}_{h} \mathrm{~d} \mathbf{x} \mathrm{d} t \\
& -\int_{0}^{T} \sum_{e \in \mathcal{E}_{h}} \int_{e} g\left(\pi_{h} \mathbf{B}-\mathbf{B}\right)_{e} \cdot\left[\boldsymbol{\varphi}_{h}\right]_{e} \mathrm{~d} \Gamma \mathrm{d} t \\
& +\int_{0}^{T} \int_{\Omega} \mathcal{C}\left(\pi_{h} \mathbf{B}-\mathbf{B}\right) \cdot \boldsymbol{\varphi}_{h} \mathrm{~d} \mathbf{x} \mathrm{d} t
\end{aligned}
$$

Case where $\mathbf{B}_{h} \in V_{h}^{m}$

$\overline{\text { From the definition of }}_{h}$ for the term (22) we have

$$
\int_{0}^{T} \int_{\Omega}\left(\pi_{h}\left(\partial_{t} \mathbf{B}\right)-\partial_{t} \mathbf{B}\right) \cdot \varphi_{h} \mathrm{~d} \mathbf{x} \mathrm{d} t=0
$$


Now we define a new projection operator $P_{h}$ by

$$
\left(P_{h} g\right)_{\left.\right|_{K}}=P_{K} g=\frac{1}{|K|} \int_{K} g \mathrm{~d} \mathbf{x}, \quad \forall g \in L^{1}(\Omega) \cap W^{1, \infty}(\Omega), \quad \text { and } \quad P_{h}=\sum_{K \in \mathcal{T}_{h}} P_{K} \mathbb{1}_{K}
$$

Then by Taylor expansion it can be easily shown that there exists a constant $C$ independent of $h$ such that

$$
\left\|P_{h} g-g\right\|_{L^{\infty}(\Omega)} \leq C h\|g\|_{W^{1, \infty}(\Omega)} .
$$

Then the $i$-th term of (23) can be recasted in

$$
\begin{aligned}
& -\int_{0}^{T} \int_{\Omega} \mathcal{A}_{i}\left(\pi_{h} \mathbf{B}-\mathbf{B}\right) \cdot \partial_{i} \boldsymbol{\varphi}_{h} \mathrm{~d} \mathbf{x} \mathrm{d} t=\int_{0}^{T} \int_{\Omega}\left(P_{h} \mathcal{A}_{i}-\mathcal{A}_{i}\right)\left(\pi_{h} \mathbf{B}-\mathbf{B}\right) \cdot \partial_{i} \varphi_{h} \mathrm{~d} \mathbf{x} \mathrm{d} t \\
& -\int_{0}^{T} \int_{\Omega}\left(\pi_{h} \mathbf{B}-\mathbf{B}\right) \cdot P_{h} \mathcal{A}_{i} \partial_{i} \boldsymbol{\varphi}_{h} \mathrm{~d} \mathbf{x} \mathrm{d} t=\int_{0}^{T} \int_{\Omega}\left(P_{h} \mathcal{A}_{i}-\mathcal{A}_{i}\right)\left(\pi_{h} \mathbf{B}-\mathbf{B}\right) \cdot \partial_{i} \boldsymbol{\varphi}_{h} \mathrm{~d} \mathbf{x} \mathrm{d} t
\end{aligned}
$$

because $\mathcal{A}_{i}$ is symmetric, $P_{h} \mathcal{A}_{i} \partial_{i} \boldsymbol{\varphi}_{h}=P_{h} u_{i} \partial_{i} \boldsymbol{\varphi}_{h} \in V_{h}^{m}(\Omega)$ and because of the definition of $\pi_{h}$. Now we give an estimate for the term (28). Thanks the approximation properties (7) and (27), the inverse properties (8), and the Cauchy-Schwarz inequality we obtain

$$
\begin{aligned}
& \left|\int_{0}^{T} \int_{\Omega}\left(P_{h} \mathcal{A}_{i}-\mathcal{A}_{i}\right)\left(\pi_{h} \mathbf{B}-\mathbf{B}\right) \cdot \partial_{i} \boldsymbol{\varphi}_{h} \mathrm{~d} \mathbf{x} \mathrm{d} t\right| \\
& \leq \int_{0}^{T} \sum_{K \in \mathcal{T}_{h}}\left(\int_{K}\left\|\pi_{h} \mathbf{B}-\mathbf{B}\right\|_{2}^{2} \mathrm{~d} \mathbf{x}\right)^{1 / 2}\left(\int_{K}\left\|\left(P_{h} \mathcal{A}_{i}-\mathcal{A}_{i}\right) \partial_{i} \boldsymbol{\varphi}_{h}\right\|_{2}^{2} \mathrm{~d} \mathbf{x}\right)^{1 / 2} \mathrm{~d} t \\
& \leq C\left(T,\left\|\mathcal{A}_{i}\right\|_{L^{\infty}\left(0, T ; W^{1, \infty}(\Omega)\right)},|\mathbf{B}|_{L^{\infty}\left(0, T ; \mathbf{H}^{m+1}(\Omega)\right)}\right) h^{m+1} \int_{0}^{T}\left\|\boldsymbol{\varphi}_{h}\right\|_{\mathbf{L}^{2}(\Omega)} \mathrm{d} t .
\end{aligned}
$$

For the term (25) we get

$$
\begin{aligned}
\left|\int_{0}^{T} \int_{\Omega} \mathcal{C}\left(\pi_{h} \mathbf{B}-\mathbf{B}\right) \cdot \boldsymbol{\varphi}_{h} \mathrm{~d} \mathbf{x} \mathrm{d} t\right| & \leq\left|\int_{0}^{T} \sum_{K \in \mathcal{T}_{h}} \int_{K} \mathcal{C}\left(\pi_{h} \mathbf{B}-\mathbf{B}\right) \cdot \boldsymbol{\varphi}_{h} \mathrm{~d} \mathbf{x} \mathrm{d} t\right| \\
& \leq\|\mathcal{C}\|_{L^{\infty}\left(0, T ; L^{\infty}(\Omega)\right)} \int_{0}^{T} \sum_{K \in \mathcal{T}_{h}}\left(\int_{K}\left\|\pi_{h} \mathbf{B}-\mathbf{B}\right\|_{2}^{2} \mathrm{~d} \mathbf{x}\right)^{1 / 2}\left\|\boldsymbol{\varphi}_{h}\right\|_{\mathbf{L}^{2}(K)} \mathrm{d} t \\
& \leq C\left(T,\|\mathcal{C}\|_{L^{\infty}\left(0, T ; L^{\infty}(\Omega)\right)},|\mathbf{B}|_{L^{\infty}\left(0, T ; \mathbf{H}^{m+1}(\Omega)\right)}\right) h^{m+1} \int_{0}^{T}\left\|\boldsymbol{\varphi}_{h}\right\|_{\mathbf{L}^{2}(\Omega)} \mathrm{d} t
\end{aligned}
$$

Now it remains to estimate the term (24). First have

$$
\begin{aligned}
g\left(\pi_{h} \mathbf{B}-\mathbf{B}\right)_{e} & =\mathcal{A}_{e, K} \overline{\left(\pi_{h} \mathbf{B}-\mathbf{B}\right)}-\frac{1}{2}\left|\mathcal{A}_{e, K}\right|\left[\left(\pi_{h} \mathbf{B}-\mathbf{B}\right)\right]_{e} \\
& =\frac{\mathcal{A}_{e, K}-\left|\mathcal{A}_{e, K}\right|}{2}\left(\pi_{h} \mathbf{B}-\mathbf{B}\right)_{K_{e}}+\frac{\mathcal{A}_{e, K}+\left|\mathcal{A}_{e, K}\right|}{2}\left(\pi_{h} \mathbf{B}-\mathbf{B}\right)_{K} \\
& \leq\left|\mathcal{A}_{e, K}\right|\left(\left|\pi_{h} \mathbf{B}-\mathbf{B}\right|_{K_{e}}+\left|\pi_{h} \mathbf{B}-\mathbf{B}\right|_{K}\right)
\end{aligned}
$$


Since $\left|\mathcal{A}_{e, K}\right|$ is symmetric, using a Young inequality we obtain

$$
\begin{gathered}
\int_{0}^{T} \sum_{e \in \mathcal{E}_{h}} \int_{e} g\left(\pi_{h} \mathbf{B}-\mathbf{B}\right)_{e} \cdot\left[\boldsymbol{\varphi}_{h}\right]_{e} \mathrm{~d} \Gamma \mathrm{d} t \leq \int_{0}^{T} \sum_{e \in \mathcal{E}_{h}} \int_{e}\left|\mathcal{A}_{e, K}\right|^{-1 / 2} g\left(\pi_{h} \mathbf{B}-\mathbf{B}\right)_{e} \cdot\left|\mathcal{A}_{e, K}\right|^{1 / 2}\left[\boldsymbol{\varphi}_{h}\right]_{e} \mathrm{~d} \Gamma \mathrm{d} t \\
\leq \int_{0}^{T} \sum_{e \in \mathcal{E}_{h}} \int_{e}\left\|\left|\mathcal{A}_{e, K}\right|^{-1 / 2} g\left(\pi_{h} \mathbf{B}-\mathbf{B}\right)_{e}\right\|_{2}^{2} \mathrm{~d} \mathbf{x} \mathrm{d} t+\frac{1}{4} \int_{0}^{T} \sum_{e \in \mathcal{E}_{h}} \int_{e}\left|\mathcal{A}_{e}\right|\left[\boldsymbol{\varphi}_{h}\right]_{e} \cdot\left[\boldsymbol{\varphi}_{h}\right]_{e} \mathrm{~d} \Gamma \mathrm{d} t .
\end{gathered}
$$

Now we want to estimate the first term of (31). Using (9) and the trace result $\left\|\gamma_{0} \mathbf{v}\right\|_{\mathbf{L}^{2}(\partial K)} \leq C\|\mathbf{v}\|_{\mathbf{H}^{1 / 2}(K)}$ we get

$$
\begin{gathered}
\int_{0}^{T} \sum_{e \in \mathcal{E}_{h}} \int_{e}\left\|\left|\mathcal{A}_{e, K}\right|^{-1 / 2} g\left(\pi_{h} \mathbf{B}-\mathbf{B}\right)_{e}\right\|_{2}^{2} \mathrm{~d} \mathbf{x} \mathrm{d} t \leq 2\|\mathbf{u}\|_{L^{\infty}\left(0, T ; L^{\infty}(\Omega)\right)} \int_{0}^{T} \sum_{K \in \mathcal{T}_{h}}\left\|\pi_{h} \mathbf{B}-\mathbf{B}\right\|_{\mathbf{L}^{2}(\partial K)}^{2} \mathrm{~d} t \\
\leq 2\|\mathbf{u}\|_{L^{\infty}\left(0, T ; L^{\infty}(\Omega)\right)} \int_{0}^{T} \sum_{K \in \mathcal{T}_{h}}\left\|\pi_{h} \mathbf{B}-\mathbf{B}\right\|_{\mathbf{H}^{1 / 2}(K)}^{2} \mathrm{~d} t \leq C\left(T,\|\mathbf{u}\|_{L^{\infty}\left(0, T ; L^{\infty}(\Omega)\right)},|\mathbf{B}|_{L^{\infty}\left(0, T ; \mathbf{H}^{m+1}(\Omega)\right)}\right) h^{2 m+1} .
\end{gathered}
$$

Finally, from (20)-(26), (29)-(32) we get

$$
\begin{aligned}
& \left\|\pi_{h} \mathbf{e}_{h}(T)\right\|_{\mathbf{L}^{2}(\Omega)}^{2}-\left\|\pi_{h} \mathbf{e}_{h}(0)\right\|_{\mathbf{L}^{2}(\Omega)}^{2}+\frac{1}{2} \int_{0}^{T} \sum_{e \in \mathcal{E}_{h}} \int_{e}\left|\mathcal{A}_{e}\right|\left[\pi_{h} \mathbf{e}_{h}\right]_{e} \cdot\left[\pi_{h} \mathbf{e}_{h}\right]_{e} \mathrm{~d} \Gamma \mathrm{d} t \leq C_{1} h^{2 m+1} \\
& \quad+C_{2} h^{m+1} \int_{0}^{T}\left\|\pi_{h} \mathbf{e}_{h}(t)\right\|_{\mathbf{L}^{2}(\Omega)}+\int_{0}^{T}\left(2\|\mathcal{C}\|_{L^{\infty}(\Omega)}+\|\nabla \cdot \mathbf{u}\|_{L^{\infty}(\Omega)}\right)\left\|\pi_{h} \mathbf{e}_{h}(t)\right\|_{\mathbf{L}^{2}(\Omega)}^{2} \mathrm{~d} t .
\end{aligned}
$$

Using a Young inequality, followed by a Gronwall inequality, from (33) we get, $\forall t \leq T$,

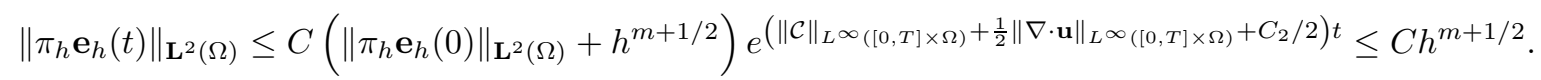

Finally we get, $\forall t \leq T$,

$$
\begin{aligned}
\left\|\mathbf{e}_{h}(t)\right\|_{\mathbf{L}^{2}(\Omega)} & \leq\left\|\pi_{h} \mathbf{e}_{h}(t)\right\|_{\mathbf{L}^{2}(\Omega)}+\left\|\pi_{h} \mathbf{e}_{h}(t)-\mathbf{e}_{h}(t)\right\|_{\mathbf{L}^{2}(\Omega)} \\
& \leq\left\|\pi_{h} \mathbf{e}_{h}(t)\right\|_{\mathbf{L}^{2}(\Omega)}+\left\|\pi_{h} \mathbf{B}(t)-\mathbf{B}(t)\right\|_{\mathbf{L}^{2}(\Omega)} \leq C h^{m+1 / 2}
\end{aligned}
$$

which finishes the proof when $\mathbf{B}_{h} \in V_{h}^{m}$.

Case where $\mathbf{B}_{h} \in W_{h}^{1}$

We have to give new estimates for the terms (22)-(25). For the term (22), using Cauchy-Schwarz inequality and the approximation property (10) we get

$$
\int_{0}^{T} \int_{\Omega}\left(\Pi_{h}\left(\partial_{t} \mathbf{B}\right)-\partial_{t} \mathbf{B}\right) \cdot \boldsymbol{\varphi}_{h} \mathrm{~d} \mathbf{x} \mathrm{d} t \leq C\left(T,\left|\partial_{t} \mathbf{B}\right|_{L^{\infty}\left(0, T ; \mathbf{H}^{1}(\Omega)\right)}\right) h \int_{0}^{T}\left\|\boldsymbol{\varphi}_{h}\right\|_{\mathbf{L}^{2}(\Omega)} \mathrm{d} t
$$

Now let us estimate the term (24). The inequality (31) is still valid. It remains to give a new estimate for the first term of the right hand side of the inequality (31). Using the approximation properties (10)-(12), and the 
fact that $\operatorname{div} \Pi_{h} \mathbf{B}=\operatorname{div} \mathbf{B}=0$ then we get

$$
\begin{aligned}
\int_{0}^{T} \sum_{e \in \mathcal{E}_{h}} \int_{e} & \left\|\left|\mathcal{A}_{e, K}\right|^{-1 / 2} g\left(\Pi_{h} \mathbf{B}-\mathbf{B}\right)_{e}\right\|_{2}^{2} \mathrm{~d} \mathbf{x} \mathrm{d} t \leq 2\|\mathbf{u}\|_{L^{\infty}\left(0, T ; L^{\infty}(\Omega)\right)} \int_{0}^{T} \sum_{K \in \mathcal{T}_{h}}\left\|\Pi_{h} \mathbf{B}-\mathbf{B}\right\|_{\mathbf{L}^{2}(\partial K)}^{2} \mathrm{~d} t \\
& \leq 2\|\mathbf{u}\|_{L^{\infty}\left(0, T ; L^{\infty}(\Omega)\right)} \int_{0}^{T} \sum_{K \in \mathcal{T}_{h}}\left\|\Pi_{h} \mathbf{B}-\mathbf{B}\right\|_{\mathcal{W}(K)}^{2} \mathrm{~d} t \\
& \leq 2\|\mathbf{u}\|_{L^{\infty}\left(0, T ; L^{\infty}(\Omega)\right)} \int_{0}^{T} \sum_{K \in \mathcal{T}_{h}}\left(\left\|\Pi_{h} \mathbf{B}-\mathbf{B}\right\|_{\mathbf{H}(\operatorname{curl} ; K)}^{2}+\left\|\left(\Pi_{h} \mathbf{B}-\mathbf{B}\right) \times \boldsymbol{\nu}\right\|_{L^{2}(\partial K)}^{2}\right) \mathrm{d} t \\
& \leq C\left(T,\|\mathbf{u}\|_{L^{\infty}\left(0, T ; L^{\infty}(\Omega)\right)},\|\mathbf{B}\|_{L^{\infty}\left(0, T ; \mathbf{H}^{2}(\Omega)\right)}\right) h^{2} .
\end{aligned}
$$

Now we give a new estimate for (23) and (25). Using the Green formula (14) we obtain

$$
\begin{aligned}
-\sum_{i=1}^{2} & \int_{0}^{T} \int_{\Omega} \mathcal{A}_{i}\left(\Pi_{h} \mathbf{B}-\mathbf{B}\right) \cdot \partial_{i} \boldsymbol{\varphi}_{h} \mathrm{~d} \mathbf{x} \mathrm{d} t+\int_{0}^{T} \int_{\Omega} \mathcal{C}\left(\Pi_{h} \mathbf{B}-\mathbf{B}\right) \cdot \boldsymbol{\varphi}_{h} \mathrm{~d} \mathbf{x} \mathrm{d} t \\
& =\int_{0}^{T} \sum_{K \in \mathcal{T}_{h}} \int_{K}\left(\sum_{i=1}^{2} \partial_{i}\left(\mathcal{A}_{i}\left(\Pi_{h} \mathbf{B}-\mathbf{B}\right)\right)+\mathcal{C}\left(\Pi_{h} \mathbf{B}-\mathbf{B}\right)\right) \cdot \boldsymbol{\varphi}_{h} \mathrm{~d} \mathbf{x} \mathrm{d} t \\
& -\int_{0}^{T} \sum_{K \in \mathcal{T}_{h}} \sum_{e \in \partial K} \int_{e} \mathcal{A}_{e, K}\left(\Pi_{h} \mathbf{B}-\mathbf{B}\right) \cdot \boldsymbol{\varphi}_{h} \mathrm{~d} \Gamma \mathrm{d} t .
\end{aligned}
$$

First we look at the term (37). Using the approximation properties (10)-(12), inverse properties (8), the fact that $\operatorname{div} \Pi_{h} \mathbf{B}=\operatorname{div} \mathbf{B}=0$ on $K$ and the Cauchy-Schwarz inequality we get

$$
\begin{aligned}
& \int_{0}^{T} \sum_{K \in \mathcal{T}_{h}} \sum_{e \in \partial K} \int_{e} \mathcal{A}_{e, K}\left(\Pi_{h} \mathbf{B}-\mathbf{B}\right) \cdot \boldsymbol{\varphi}_{h} \mathrm{~d} \Gamma \mathrm{d} t \leq 2\|\mathbf{u}\|_{L^{\infty}\left(0, T ; L^{\infty}(\Omega)\right)} \int_{0}^{T} \sum_{K \in \mathcal{T}_{h}}\left\|\Pi_{h} \mathbf{B}-\mathbf{B}\right\|_{\mathbf{L}^{2}(\partial K)}\left\|\boldsymbol{\varphi}_{h}\right\|_{\mathbf{L}^{2}(\partial K)} \mathrm{d} t \\
\leq & 2\|\mathbf{u}\|_{L^{\infty}\left(0, T ; L^{\infty}(\Omega)\right)} \int_{0}^{T} \sum_{K \in \mathcal{T}_{h}} h^{-1 / 2}\left\|\Pi_{h} \mathbf{B}-\mathbf{B}\right\|_{\mathcal{W}(K)}\left\|\boldsymbol{\varphi}_{h}\right\|_{\mathbf{L}^{2}(K)} \mathrm{d} t \\
\leq & 2\|\mathbf{u}\|_{L^{\infty}\left(0, T ; L^{\infty}(\Omega)\right)} \int_{0}^{T} \sum_{K \in \mathcal{T}_{h}} h^{-1 / 2}\left(\left\|\Pi_{h} \mathbf{B}-\mathbf{B}\right\|_{\mathbf{H}(\operatorname{curl} ; K)}+\left\|\left(\Pi_{h} \mathbf{B}-\mathbf{B}\right) \times \boldsymbol{\nu}\right\|_{L^{2}(\partial K)}\right)\left\|\boldsymbol{\varphi}_{h}\right\|_{\mathbf{L}^{2}(K)} \mathrm{d} t \\
\leq & C\left(T,\|\mathbf{u}\|_{L^{\infty}\left(0, T ; L^{\infty}(\Omega)\right)},\|\mathbf{B}\|_{L^{\infty}\left(0, T ; \mathbf{H}^{2}(\Omega)\right)}\right) h^{1 / 2} \int_{0}^{T}\left\|\boldsymbol{\varphi}_{h}\right\|_{\mathbf{L}^{2}(\Omega)} \mathrm{d} t .
\end{aligned}
$$

As $\operatorname{div} \Pi_{h} \mathbf{B}=\operatorname{div} \mathbf{B}=0$ on $K$ the term (36) can be recasted in

$$
\begin{aligned}
& \int_{0}^{T} \sum_{K \in \mathcal{T}_{h}} \int_{K}\left(\sum_{i=1}^{2} \partial_{i}\left(\mathcal{A}_{i}\left(\Pi_{h} \mathbf{B}-\mathbf{B}\right)\right)+\mathcal{C}\left(\Pi_{h} \mathbf{B}-\mathbf{B}\right)\right) \cdot \boldsymbol{\varphi}_{h} \mathrm{~d} \mathbf{x} \mathrm{d} t \\
= & \int_{0}^{T} \sum_{K \in \mathcal{T}_{h}} \int_{K} \nabla \times\left(\left(\Pi_{h} \mathbf{B}-\mathbf{B}\right) \times \mathbf{u}\right) \cdot \boldsymbol{\varphi}_{h} \mathrm{~d} \mathbf{x} \mathrm{d} t \\
= & \int_{0}^{T} \sum_{K \in \mathcal{T}_{h}} \int_{K}\left\{\left(\left(\Pi_{h} \mathbf{B}-\mathbf{B}\right) \cdot \nabla\right) \mathbf{u}+\left(\Pi_{h} \mathbf{B}-\mathbf{B}\right) \operatorname{div} \mathbf{u}+(\mathbf{u} \cdot \nabla)\left(\Pi_{h} \mathbf{B}-\mathbf{B}\right)\right\} \cdot \boldsymbol{\varphi}_{h} \mathrm{~d} \mathbf{x} \mathrm{d} t .
\end{aligned}
$$


Now we have to estimates the three terms of (39). For the two first term of (39) we get

$$
\begin{gathered}
\int_{0}^{T} \sum_{K \in \mathcal{T}_{h}} \int_{K}\left(\left(\Pi_{h} \mathbf{B}-\mathbf{B}\right) \cdot \nabla\right) \mathbf{u} \cdot \boldsymbol{\varphi}_{h} \mathrm{~d} \mathbf{x} \mathrm{d} t \leq 2\|\mathbf{u}\|_{L^{\infty}\left(0, T ; W^{1, \infty}(\Omega)\right)} \int_{0}^{T} \sum_{K \in \mathcal{T}_{h}}\left\|\Pi_{h} \mathbf{B}-\mathbf{B}\right\|_{\mathbf{L}^{2}(K)}\left\|\boldsymbol{\varphi}_{h}\right\|_{\mathbf{L}^{2}(K)} \mathrm{d} t \\
\leq C\left(T,\|\mathbf{u}\|_{L^{\infty}\left(0, T ; W^{1, \infty}(\Omega)\right)},|\mathbf{B}|_{L^{\infty}\left(0, T ; \mathbf{H}^{1}(\Omega)\right)}\right) h \int_{0}^{T}\left\|\boldsymbol{\varphi}_{h}\right\|_{\mathbf{L}^{2}(\Omega)} \mathrm{d} t
\end{gathered}
$$

and

$$
\begin{gathered}
\int_{0}^{T} \sum_{K \in \mathcal{T}_{h}} \int_{K}\left(\Pi_{h} \mathbf{B}-\mathbf{B}\right) \operatorname{div} \mathbf{u} \cdot \boldsymbol{\varphi}_{h} \mathrm{~d} \mathbf{x} \mathrm{d} t \leq\|\operatorname{div} \mathbf{u}\|_{L^{\infty}\left(0, T ; L^{\infty}(\Omega)\right)} \int_{0}^{T} \sum_{K \in \mathcal{T}_{h}}\left\|\Pi_{h} \mathbf{B}-\mathbf{B}\right\|_{\mathbf{L}^{2}(K)}\left\|\boldsymbol{\varphi}_{h}\right\|_{\mathbf{L}^{2}(K)} \mathrm{d} t \\
\leq C\left(T,\|\operatorname{div} \mathbf{u}\|_{L^{\infty}\left(0, T ; L^{\infty}(\Omega)\right)},|\mathbf{B}|_{L^{\infty}\left(0, T ; \mathbf{H}^{1}(\Omega)\right)}\right) h \int_{0}^{T}\left\|\boldsymbol{\varphi}_{h}\right\|_{\mathbf{L}^{2}(\Omega)} \mathrm{d} t
\end{gathered}
$$

Using the approximation properties (10)-(12), the continuous imbedding (13), the fact that $\operatorname{div} \Pi_{h} \mathbf{B}=\operatorname{div} \mathbf{B}=0$ on $K$ and the Cauchy-Schwarz inequality then we get

$$
\begin{aligned}
& \int_{0}^{T} \sum_{K \in \mathcal{T}_{h}} \int_{K}(\mathbf{u} \cdot \nabla)\left(\Pi_{h} \mathbf{B}-\mathbf{B}\right) \cdot \boldsymbol{\varphi}_{h} \mathrm{~d} \mathbf{x} \mathrm{d} t \\
\leq & 2\|\mathbf{u}\|_{L^{\infty}\left(0, T ; L^{\infty}(\Omega)\right)} \int_{0}^{T} \sum_{K \in \mathcal{T}_{h}}\left\|\Pi_{h} \mathbf{B}-\mathbf{B}\right\|_{\mathbf{H}^{1}(K)}\left\|\boldsymbol{\varphi}_{h}\right\|_{\mathbf{L}^{2}(K)} \mathrm{d} t \\
\leq & 2\|\mathbf{u}\|_{L^{\infty}\left(0, T ; L^{\infty}(\Omega)\right)} \int_{0}^{T} \sum_{K \in \mathcal{T}_{h}}\left(\left\|\Pi_{h} \mathbf{B}-\mathbf{B}\right\|_{\mathbf{H}(\operatorname{curl} ; K)}+\sum_{e \in \partial K}\left\|\left(\Pi_{h} \mathbf{B}-\mathbf{B}\right) \times \boldsymbol{\nu}_{e}\right\|_{\mathbf{H}^{1 / 2}(e)}\right)\left\|\boldsymbol{\varphi}_{h}\right\|_{\mathbf{L}^{2}(K)} \mathrm{d} t \\
\leq & C\left(T,\|\mathbf{u}\|_{L^{\infty}\left(0, T ; L^{\infty}(\Omega)\right)},\|\mathbf{B}\|_{L^{\infty}\left(0, T ; \mathbf{H}^{2}(\Omega)\right)}\right) h^{1 / 2} \int_{0}^{T}\left\|\boldsymbol{\varphi}_{h}\right\|_{\mathbf{L}^{2}(\Omega)} \mathrm{d} t .
\end{aligned}
$$

Finally, by introducing the estimates (34)-(42) into the relations (20)-(25) and using a Young inequality followed by a Gronwall inequality we get

$$
\left\|\Pi_{h} \mathbf{e}_{h}(t)\right\|_{\mathbf{L}^{2}(\Omega)} \leq C\left(\left\|\Pi_{h} \mathbf{e}_{h}(0)\right\|_{\mathbf{L}^{2}(\Omega)}+h^{1 / 2}\right) e^{\left(\|\mathcal{C}\|_{L^{\infty}([0, T] \times \Omega)}+\frac{1}{2}\|\nabla \cdot \mathbf{u}\|_{L^{\infty}([0, T] \times \Omega)}+C_{2} / 2\right) t} \leq C h^{1 / 2}
$$

and $\forall t \leq T$, we obtain

$$
\left\|\mathbf{e}_{h}(t)\right\|_{\mathbf{L}^{2}(\Omega)} \leq\left\|\Pi_{h} \mathbf{e}_{h}(t)\right\|_{\mathbf{L}^{2}(\Omega)}+\left\|\Pi_{h} \mathbf{e}_{h}(t)-\mathbf{e}_{h}(t)\right\|_{\mathbf{L}^{2}(\Omega)}, \quad \leq C h^{1 / 2}
$$

which ends the proof when $\mathbf{B}_{h} \in W_{h}^{1}$.

Remark 3. When $\mathbf{B}_{h} \in W_{h}^{1}$ we can not expect to obtain error estimate like $\mathcal{O}\left(h^{3 / 2}\right)$ which is got when $\mathbf{B}_{h} \in V_{h}^{1}$. The reason is that the space $\mathcal{R}_{1}$ does not reproduce all the polynomial fields of $\mathbb{P}^{1}$ which are divergence free. Then the approximation properties of $\mathcal{R}_{1}$ are the same as the ones of $\mathbb{P}^{0}$. That's the reason why we loose one order of convergence. Nevertheless we could loose one order more if there were not the approximation property (11). As the approximation in the space $\mathbf{H}$ (curl) and the space $\mathbf{L}^{2}$ is of the same order we can get convergence of the scheme whose the convergence rate is in $\mathcal{O}\left(h^{1 / 2}\right)$, and that's why the Nédélec space is interesting. Unfortunately it could not be generalized to the space $\mathcal{R}_{k}$ with $k>1$ as div $\mathcal{R}_{k} \neq 0$. We observe that $\mathbb{P}^{0} \subset \mathcal{R}_{1} \subset \mathbb{P}^{1}$ and that $W_{h}^{1} \subset V_{h}^{1}$ with $\operatorname{dim}\left(W_{h}^{1}\right)=3$ and $\operatorname{dim}\left(V_{h}^{1}\right)=5$. 


\section{Analysis of the Fully Discretized scheme}

In this section we present the stability, convergence and error analysis in $L^{2}$ of the fully discretized scheme. The main result is

Theorem 5. Let $\mathbf{u}$ and $\mathbf{B}_{0}$ sufficiently regular, typically we consider that $\mathbf{u} \in W^{1, \infty}\left(\left[0,+\infty\left[\times\left(\mathbb{R}^{2}\right)\right)\right.\right.$ and $\mathbf{B}_{0} \in \mathbf{H}^{m+1}\left(\mathbb{R}^{2}\right)$ and let us assume that there exists a constant $\beta(\alpha)$ depending on $\alpha$ such that the $C F L$ condition $\Delta t \leq \beta(\alpha) h^{4 / 3}$ holds. Moreover we assume the condition (3) and $\left\|\delta_{\mathbf{B}}^{0}\right\|_{\mathbf{L}^{2}(\Omega)}=\left\|\mathbf{B}^{0}-\pi_{h} \mathbf{B}^{0}\right\|_{\mathbf{L}^{2}(\Omega)}=\mathcal{O}\left(h^{m+1}\right)$. Then there exists a constant $C=C\left(\|\mathbf{u}\|_{W^{1, \infty}([0, T] \times \Omega)}, T, \alpha\right)$ independent of $h$ such that

$$
\left\|\mathbf{B}_{h}\right\|_{l \infty\left(0, T ; \mathbf{L}^{2}(\Omega)\right)} \leq C\left\|\mathbf{B}_{h}(0)\right\|_{\mathbf{L}^{2}(\Omega)},
$$

where $\mathbf{B}_{h}(0) \in V_{h}^{m}$ or $\mathbf{B}_{h}(0) \in W_{h}^{m}$ with $m=1$. Moreover there exists a constant $C=C\left(T, \alpha,\|\mathbf{u}\|_{W^{1, \infty}([0, T] \times \Omega)}\right.$, $\left.\|\mathbf{B}\|_{W^{3, \infty}\left(0, T ; \mathbf{H}^{m+1}\left(\mathbb{R}^{2}\right)\right)}\right)$ independent of $h$ such that

$$
\left\|\mathbf{B}-\mathbf{B}_{h}\right\|_{l^{\infty}\left(0, T ; \mathbf{L}^{2}(\Omega)\right)}+\sqrt{\Delta t \sum_{n=0}^{N_{T}} \sum_{e \in \mathcal{E}_{h}} \int_{e}\left|\mathcal{A}_{e}^{n}\right|\left[\mathbf{B}_{h}^{n}-\mathbf{B}\left(t^{n}\right)\right]_{e} \cdot\left[\mathbf{B}_{h}^{n}-\mathbf{B}\left(t^{n}\right)\right]_{e} \mathrm{~d} \Gamma} \leq C\left(\Delta t^{2}+h^{m+\eta}\right)
$$

where $\eta=\frac{1}{2}$ if $\mathbf{B}_{h} \in V_{h}^{m}$ and $\eta=-\frac{1}{2}$ if $\mathbf{B}_{h} \in W_{h}^{m}$ with $m=1$.

Remark 4. The CFL condition $\Delta t \leq \beta(\alpha) h^{4 / 3}$ is surprising, but it seems that it is linked to the time discretization with a Runge-Kutta scheme of order two. Indeed in [23] the author also obtains the convergence of a second order Runge-Kutta finite element scheme under the same CFL condition. The minimal regularity assumptions to obtain convergence are $\mathbf{u} \in W^{1, \infty}$ and $\mathbf{B}_{0} \in \mathbf{H}^{1}$ when $\mathbf{B}_{h} \in V_{h}^{0}$ or $\mathbf{B}_{0} \in \mathbf{H}^{1}(\operatorname{curl})$ when $\mathbf{B}_{h} \in W_{h}^{1}$

Proof.

$L^{2}$-stability. We begin by proving the theorem when $\mathbf{B}_{h} \in V_{h}^{m}$ and therefore we will modify some estimates to adapt the proof when $\mathbf{B}_{h} \in W_{h}^{1}$. First we begin by proving the $L^{2}$-stability of the scheme. This proof remains true for the two choices of approximation space. If we take $\boldsymbol{\varphi}_{h}=\mathbf{B}_{h}^{n}$ in (16), and $\boldsymbol{\varphi}_{h}=\mathbf{Y}_{h}^{n}$ in (17) then after summing over all elements $K$ of the partition $\mathcal{T}_{h}$ the sum $(16) / 2+(17)$ gives

$$
\left\|\mathbf{B}_{h}^{n+1}\right\|_{\mathbf{L}^{2}(\Omega)}^{2}-\left\|\mathbf{B}_{h}^{n}\right\|_{\mathbf{L}^{2}(\Omega)}^{2}-\left\|\mathbf{B}_{h}^{n+1}-\mathbf{Y}_{h}^{n}\right\|_{\mathbf{L}^{2}(\Omega)}^{2}=\Delta t\left(\mathcal{F}^{n}\left(\mathbf{B}_{h}^{n}, \mathbf{B}_{h}^{n}\right)+\mathcal{F}^{n+1}\left(\mathbf{Y}_{h}^{n}, \mathbf{Y}_{h}^{n}\right)\right) .
$$

where $\mathcal{F}^{n}=\sum_{K \in \mathcal{T}_{h}} \mathcal{F}_{K}^{n} \mathbb{1}_{K}$. First we will give an estimate of terms of the form of $\mathcal{F}^{n}\left(\boldsymbol{\varphi}_{h}, \boldsymbol{\varphi}_{h}\right)$ and in the second moment we will give an estimate for the term $\left\|\mathbf{B}_{h}^{n+1}-\mathbf{Y}_{h}^{n}\right\|_{\mathbf{L}^{2}(\Omega)}$. Using (129) and the Green formula (14) we get

$$
\mathcal{F}^{n}\left(\boldsymbol{\varphi}_{h}, \boldsymbol{\varphi}_{h}\right)=-\frac{1}{2} \sum_{e \in \mathcal{E}_{h}} \int_{e}\left|\mathcal{A}_{e, K}^{n}\right|\left[\boldsymbol{\varphi}_{h}\right]_{e} \cdot\left[\boldsymbol{\varphi}_{h}\right]_{e} \mathrm{~d} \Gamma-\int_{\Omega} \mathcal{C}^{n} \boldsymbol{\varphi}_{h} \cdot \boldsymbol{\varphi}_{h} \mathrm{~d} \mathbf{x} \mathrm{d} t-\frac{1}{2} \int_{\Omega} \nabla \cdot \mathbf{u}^{n}\left\|\boldsymbol{\varphi}_{h}\right\|_{2}^{2} \mathrm{~d} \mathbf{x} .
$$

From the condition (3), and the equations (44) and (43) we get

$$
\left\|\mathbf{B}_{h}^{n+1}\right\|_{\mathbf{L}^{2}(\Omega)}^{2} \leq\left(1-\alpha \frac{\Delta t}{2}\right)\left\|\mathbf{B}_{h}^{n}\right\|_{\mathbf{L}^{2}(\Omega)}^{2}-\alpha \frac{\Delta t}{2}\left\|\mathbf{Y}_{h}^{n}\right\|_{\mathbf{L}^{2}(\Omega)}^{2}+\left\|\mathbf{B}_{h}^{n+1}-\mathbf{Y}_{h}^{n}\right\|_{\mathbf{L}^{2}(\Omega)}^{2} .
$$

It remains to estimate the term $\left\|\mathbf{B}_{h}^{n+1}-\mathbf{Y}_{h}^{n}\right\|_{\mathbf{L}^{2}(\Omega)}$. To do this we remark that after summing over all elements $K$ of the partition $\mathcal{T}_{h}$ the sum (17)-(16)/2 gives

$$
\int_{\Omega}\left(\mathbf{B}_{h}^{n+1}-\mathbf{Y}_{h}^{n}\right) \cdot \boldsymbol{\varphi}_{h} \mathrm{~d} \mathbf{x}=\frac{\Delta t}{2}\left(\mathcal{F}^{n+1}\left(\mathbf{Y}_{h}^{n}, \boldsymbol{\varphi}_{h}\right)-\mathcal{F}^{n}\left(\mathbf{B}_{h}^{n}, \boldsymbol{\varphi}_{h}\right)\right)
$$


Let us estimate the right hand side of (46). We have

$$
\begin{aligned}
& \frac{\Delta t}{2}\left(\mathcal{F}^{n+1}\left(\mathbf{Y}_{h}^{n}, \boldsymbol{\varphi}_{h}\right)-\mathcal{F}^{n}\left(\mathbf{B}_{h}^{n}, \boldsymbol{\varphi}_{h}\right)\right)=\sum_{K \in \mathcal{T}_{h}} \sum_{i=1}^{2} \int_{K} \Delta t\left(\mathcal{A}_{i}^{n+1} \mathbf{Y}_{h}^{n}-\mathcal{A}_{i}^{n} \mathbf{B}_{h}^{n}\right) \cdot \partial_{i} \boldsymbol{\varphi}_{h} \mathrm{~d} \mathbf{x} \\
- & \sum_{K \in \mathcal{T}_{h}} \sum_{e \in \partial K} \int_{e} \Delta t\left(g^{n+1}\left(\mathbf{Y}_{h}^{n}\right)_{e}-g^{n}\left(\mathbf{B}_{h}^{n}\right)_{e}\right) \cdot \boldsymbol{\varphi}_{K} \mathrm{~d} \mathbf{x}-\sum_{K \in \mathcal{T}_{h}} \int_{K} \Delta t\left(\mathcal{C}^{n+1} \mathbf{Y}_{h}^{n}-\mathcal{C}^{n} \mathbf{B}_{h}^{n}\right) \cdot \partial_{i} \boldsymbol{\varphi}_{h} \mathrm{~d} \mathbf{x} .
\end{aligned}
$$

By seeing that there exists a time $t^{\natural}$ and $t^{\dagger}$ such that $\mathcal{A}_{e, K}^{n}=\mathcal{A}_{e, K}^{n+1}-\Delta t \partial_{t} \mathcal{A}_{e, K}^{\natural}$ and $\left|\mathcal{A}_{e, K}^{n}\right|=\left|\mathcal{A}_{e, K}^{n+1}\right|-\Delta t \partial_{t}\left|\mathcal{A}_{e, K}^{\dagger}\right|$ where $t^{\natural}=t^{n}+\theta^{\natural} \Delta t$ and $t^{\dagger}=t^{n}+\theta^{\dagger} \Delta t$, with $0 \leq \theta^{\natural} \leq 1$ and $0 \leq \theta^{\dagger} \leq 1$, then we get

$$
\begin{aligned}
g^{n+1}\left(\mathbf{Y}_{h}^{n}\right)_{e}-g^{n}\left(\mathbf{B}_{h}^{n}\right)_{e} & =\mathcal{A}_{e, K}^{n+1} \overline{\mathbf{Y}}_{h}^{n}-\frac{\left|\mathcal{A}_{e, K}^{n+1}\right|}{2}\left[\mathbf{Y}_{h}^{n}\right]_{e}-\mathcal{A}_{e, K}^{n} \overline{\mathbf{B}}_{h}^{n}+\frac{\left|\mathcal{A}_{e, K}^{n}\right|}{2}\left[\mathbf{B}_{h}^{n}\right]_{e} \\
& =-g^{n+1}\left(\mathbf{B}_{h}^{n}-\mathbf{Y}_{h}^{n}\right)_{e}+\Delta t g^{\sharp}\left(\mathbf{B}_{h}^{n}\right)_{e}
\end{aligned}
$$

where $g^{\sharp}\left(\mathbf{B}_{h}^{n}\right)_{e}=\partial_{t} \mathcal{A}_{e, K}^{\natural} \overline{\mathbf{B}}_{h}^{n}-\frac{\partial_{t}\left|\mathcal{A}_{e, K}^{\dagger}\right|}{2}\left[\mathbf{B}_{h}^{n}\right]_{e}$. Then (47) can be rewritten as

$$
\frac{\Delta t}{2}\left(\mathcal{F}^{n+1}\left(\mathbf{Y}_{h}^{n}, \boldsymbol{\varphi}_{h}\right)-\mathcal{F}^{n}\left(\mathbf{B}_{h}^{n}, \boldsymbol{\varphi}_{h}\right)\right)=-\frac{\Delta t}{2} \mathcal{F}^{n+1}\left(\mathbf{B}_{h}^{n}-\mathbf{Y}_{h}^{n}, \boldsymbol{\varphi}_{h}\right)+\mathcal{G}\left(\mathbf{B}_{h}^{n}, \boldsymbol{\varphi}_{h}\right)
$$

where

$$
\mathcal{G}\left(\mathbf{B}_{h}^{n}, \boldsymbol{\varphi}_{h}\right)=\Delta t^{2} \sum_{i=1}^{2} \int_{\Omega} \partial_{t} \mathcal{A}_{i}^{\ddagger i} \mathbf{B}_{h}^{n} \cdot \partial_{i} \boldsymbol{\varphi}_{h} \mathrm{~d} \mathbf{x}-\Delta t^{2} \sum_{K \in \mathcal{T}_{h}} \sum_{e \in \partial K} \int_{e} g^{\sharp}\left(\mathbf{B}_{h}^{n}\right)_{e} \cdot \boldsymbol{\varphi}_{K} \mathrm{~d} \Gamma-\Delta t^{2} \int_{\Omega} \partial_{t} \mathcal{C}^{b} \mathbf{B}_{h}^{n} \cdot \boldsymbol{\varphi}_{h} \mathrm{~d} \mathbf{x}
$$

with $t^{\ddagger i}=t^{n}+\theta^{\ddagger i} \Delta t$ and $t^{b}=t^{n}+\theta^{b} \Delta t$ such that $\mathcal{A}_{i}^{n}=\mathcal{A}_{i}^{n+1}-\Delta t \partial_{t} \mathcal{A}_{i}^{\ddagger i}$ and $\mathcal{C}^{n}=\mathcal{C}^{n+1}-\Delta t \partial_{t} \mathcal{C}^{b}$. First we estimate the term $\mathcal{G}\left(\mathbf{B}_{h}^{n}, \boldsymbol{\varphi}_{h}\right)$. From the Young and Cauchy-Schwarz inequalities and the inverse properties (8) we get

$$
\begin{aligned}
\left|\Delta t^{2} \sum_{K \in \mathcal{T}_{h}} \int_{K} \partial_{t} \mathcal{A}_{i}^{\ddagger i} \mathbf{B}_{h}^{n} \cdot \partial_{i} \boldsymbol{\varphi}_{h} \mathrm{~d} \mathbf{x}\right| & \leq \sum_{K \in \mathcal{T}_{h}} \int_{K}\left\{C(\varepsilon) \frac{\Delta t^{4}}{h^{2}}\left\|\partial_{t} \mathcal{A}_{i}^{\ddagger i}\right\|_{L^{\infty}\left([0, T] \times \mathbb{R}^{2}\right)}\left\|\mathbf{B}_{h}^{n}\right\|_{2}^{2}+\frac{\varepsilon}{3} h^{2}\left\|\partial_{i} \boldsymbol{\varphi}_{h}\right\|_{2}^{2}\right\} \mathrm{d} \mathbf{x} \\
& \leq C(\varepsilon) \frac{\Delta t^{4}}{h^{2}}\left\|\mathbf{B}_{h}^{n}\right\|_{\mathbf{L}^{2}(\Omega)}^{2}+\frac{\varepsilon}{3}\left\|\boldsymbol{\varphi}_{h}\right\|_{\mathbf{L}^{2}(\Omega)}^{2},
\end{aligned}
$$

$$
\left|\Delta t^{2} \sum_{K \in \mathcal{T}_{h}} \int_{K} \partial_{t} \mathcal{C}^{b} \mathbf{B}_{h}^{n} \cdot \boldsymbol{\varphi}_{h} \mathrm{~d} \mathbf{x}\right| \leq C\left(\left\|\partial_{t} \mathcal{C}^{b}\right\|_{L^{\infty}\left([0, T] \times \mathbb{R}^{2}\right)}, \varepsilon\right) \Delta t^{4}\left\|\mathbf{B}_{h}^{n}\right\|_{\mathbf{L}^{2}(\Omega)}^{2}+\frac{\varepsilon}{3}\left\|\boldsymbol{\varphi}_{h}\right\|_{\mathbf{L}^{2}(\Omega)}^{2},
$$


and

$$
\begin{aligned}
& \left|\Delta t^{2} \sum_{K \in \mathcal{T}_{h}} \sum_{e \in \partial K} \int_{e} g^{\sharp}\left(\mathbf{B}_{h}^{n}\right)_{e} \cdot \boldsymbol{\varphi}_{K} \mathrm{~d} \Gamma\right| \\
& \quad \leq\left|\Delta t^{2} \sum_{K \in \mathcal{T}_{h}} \sum_{e \in \partial K} \int_{e}\left(\frac{\partial_{t} \mathcal{A}_{e, K}^{\natural}-\partial_{t}\left|\mathcal{A}_{e, K}^{\dagger}\right|}{2} \mathbf{B}_{K_{e}}^{n}+\frac{\partial_{t} \mathcal{A}_{e, K}^{\natural}+\partial_{t}\left|\mathcal{A}_{e, K}^{\dagger}\right|}{2} \mathbf{B}_{K}^{n}\right) \cdot \boldsymbol{\varphi}_{K} \mathrm{~d} \Gamma\right| \\
& \quad \leq C\left(\left\|\partial_{t} \mathcal{A}_{e, K}\right\|_{L^{\infty}\left([0, T] \times \mathbb{R}^{2}\right)}\right) \Delta t^{2} \sum_{K \in \mathcal{T}_{h}} \sum_{e \in \partial K} \int_{e}\left(\left\|\mathbf{B}_{K_{e}}^{n}\right\|_{2}\left\|\boldsymbol{\varphi}_{K}\right\|_{2}+\left\|\mathbf{B}_{K}^{n}\right\|_{2}\left\|\boldsymbol{\varphi}_{K}\right\|_{2}\right) \mathrm{d} \Gamma \\
& \quad \leq C(\varepsilon) \frac{\Delta t^{4}}{h} \sum_{K \in \mathcal{T}_{h}}\left\|\mathbf{B}_{h}^{n}\right\|_{\mathbf{L}^{2}(\partial K)}^{2}+h \frac{\varepsilon}{3} \sum_{K \in \mathcal{T}_{h}}\left\|\boldsymbol{\varphi}_{h}\right\|_{\mathbf{L}^{2}(\partial K)}^{2} \leq C(\varepsilon) \frac{\Delta t^{4}}{h^{2}}\left\|\mathbf{B}_{h}^{n}\right\|_{\mathbf{L}^{2}(\Omega)}^{2}+\frac{\varepsilon}{3}\left\|\boldsymbol{\varphi}_{h}\right\|_{\mathbf{L}^{2}(\Omega)}^{2} .
\end{aligned}
$$

From (49)-(51), we get

$$
\left|\mathcal{G}\left(\mathbf{B}_{h}^{n}, \boldsymbol{\varphi}_{h}\right)\right| \leq C(\varepsilon)\left(\frac{\Delta t^{4}}{h^{2}}+\Delta t^{4}\right)\left\|\mathbf{B}_{h}^{n}\right\|_{\mathbf{L}^{2}(\Omega)}^{2}+\varepsilon\left\|\boldsymbol{\varphi}_{h}\right\|_{\mathbf{L}^{2}(\Omega)}^{2}
$$

Now we will estimate the term $\Delta t \mathcal{F}^{n+1}\left(\mathbf{V}_{h}, \boldsymbol{\varphi}_{h}\right)$. From the Young and Cauchy-Schwarz inequalities and the inverse properties (8) we get

$$
\begin{gathered}
\left|\Delta t \sum_{K \in \mathcal{T}_{h}} \int_{K} \mathcal{A}_{i}^{n+1} \mathbf{V}_{h} \cdot \partial_{i} \boldsymbol{\varphi}_{h} \mathrm{~d} \mathbf{x}\right| \leq C(\varepsilon) \frac{\Delta t^{2}}{h^{2}}\left\|\mathbf{V}_{h}\right\|_{\mathbf{L}^{2}(\Omega)}^{2}+\frac{\varepsilon}{3}\left\|\boldsymbol{\varphi}_{h}\right\|_{\mathbf{L}^{2}(\Omega)}^{2}, \\
\left|\Delta t \sum_{K \in \mathcal{T}_{h}} \int_{K} \mathcal{C}^{n+1} \mathbf{V}_{h} \cdot \boldsymbol{\varphi}_{h} \mathrm{~d} \mathbf{x}\right| \leq C(\varepsilon) \Delta t^{2}\left\|\mathbf{V}_{h}\right\|_{\mathbf{L}^{2}(\Omega)}^{2}+\frac{\varepsilon}{3}\left\|\boldsymbol{\varphi}_{h}\right\|_{\mathbf{L}^{2}(\Omega)}^{2},
\end{gathered}
$$

and

$$
\left|\Delta t \sum_{K \in \mathcal{T}_{h}} \sum_{e \in \partial K} \int_{e} g^{n+1}\left(\mathbf{V}_{h}\right)_{e} \cdot \boldsymbol{\varphi}_{K} \mathrm{~d} \Gamma\right| \leq C(\varepsilon) \frac{\Delta t^{2}}{h^{2}}\left\|\mathbf{V}_{h}\right\|_{\mathbf{L}^{2}(\Omega)}^{2}+\frac{\varepsilon}{3}\left\|\boldsymbol{\varphi}_{h}\right\|_{\mathbf{L}^{2}(\Omega)}^{2} .
$$

From (53)-(55), we get that

$$
\left|\Delta t \mathcal{F}\left(\mathbf{V}_{h}, \boldsymbol{\varphi}_{h}\right)\right| \leq C(\varepsilon) \frac{\Delta t^{2}}{h^{2}}\left\|\mathbf{V}_{h}\right\|_{\mathbf{L}^{2}(\Omega)}^{2}+\varepsilon\left\|\boldsymbol{\varphi}_{h}\right\|_{\mathbf{L}^{2}(\Omega)}^{2}
$$

By taking $\boldsymbol{\varphi}_{h}=\mathbf{B}_{h}^{n+1}-\mathbf{Y}_{h}^{n}$ in (46), and from (52) and (56) we obtain

$$
\begin{aligned}
& \left\|\mathbf{B}_{h}^{n+1}-\mathbf{Y}_{h}^{n}\right\|_{\mathbf{L}^{2}(\Omega)}^{2} \leq\left|\Delta t \mathcal{F}\left(\mathbf{B}_{h}^{n}-\mathbf{Y}_{h}^{n}, \mathbf{B}_{h}^{n+1}-\mathbf{Y}_{h}^{n}\right)\right|+\left|\mathcal{G}\left(\mathbf{B}_{h}^{n}, \mathbf{B}_{h}^{n+1}-\mathbf{Y}_{h}^{n}\right)\right| \\
\leq & 2 \varepsilon\left\|\mathbf{B}_{h}^{n+1}-\mathbf{Y}_{h}^{n}\right\|_{\mathbf{L}^{2}(\Omega)}^{2}+C(\varepsilon) \frac{\Delta t^{2}}{h^{2}}\left\|\mathbf{B}_{h}^{n}-\mathbf{Y}_{h}^{n}\right\|_{\mathbf{L}^{2}(\Omega)}^{2}+C(\varepsilon)\left(\frac{\Delta t^{4}}{h^{2}}+\Delta t^{4}\right)\left\|\mathbf{B}_{h}^{n}\right\|_{\mathbf{L}^{2}(\Omega)}^{2} .
\end{aligned}
$$

Let us estimate $\left\|\mathbf{B}_{h}^{n}-\mathbf{Y}_{h}^{n}\right\|_{\mathbf{L}^{2}(\Omega)}^{2}$. By taking $\boldsymbol{\varphi}_{h}=\mathbf{B}_{h}^{n}-\mathbf{Y}_{h}^{n}$ in (16) and from the estimate (56) we get

$$
\left\|\mathbf{B}_{h}^{n}-\mathbf{Y}_{h}^{n}\right\|_{\mathbf{L}^{2}(\Omega)}^{2}=\Delta t \mathcal{F}^{n}\left(\mathbf{B}_{h}^{n}, \mathbf{B}_{h}^{n}-\mathbf{Y}_{h}^{n}\right) \leq \varepsilon\left\|\mathbf{B}_{h}^{n}-\mathbf{Y}_{h}^{n}\right\|_{\mathbf{L}^{2}(\Omega)}^{2}+C(\varepsilon) \frac{\Delta t^{2}}{h^{2}}\left\|\mathbf{B}_{h}^{n}\right\|_{\mathbf{L}^{2}(\Omega)}^{2} .
$$


If $\varepsilon$ is small enough, from (58) we deduce $\left\|\mathbf{B}_{h}^{n}-\mathbf{Y}_{h}^{n}\right\|_{\mathbf{L}^{2}(\Omega)}^{2} \leq C(\varepsilon) \frac{\Delta t^{2}}{h^{2}}\left\|\mathbf{B}_{h}^{n}\right\|_{\mathbf{L}^{2}(\Omega)}^{2}$ and using (57) we get

$$
\left\|\mathbf{B}_{h}^{n+1}-\mathbf{Y}_{h}^{n}\right\|_{\mathbf{L}^{2}(\Omega)}^{2} \leq C(\varepsilon)\left(\Delta t^{4}+\frac{\Delta t^{4}}{h^{4}}+\frac{\Delta t^{4}}{h^{2}}\right)\left\|\mathbf{B}_{h}^{n}\right\|_{\mathbf{L}^{2}(\Omega)}^{2} .
$$

If we suppose $\Delta t \leq C(\varepsilon) h^{4 / 3}$, from (59) and (45) we get

$$
\left\|\mathbf{B}_{h}^{n+1}\right\|_{\mathbf{L}^{2}(\Omega)}^{2} \leq(1+C \Delta t)\left\|\mathbf{B}_{h}^{n}\right\|_{\mathbf{L}^{2}(\Omega)}^{2} \quad \text { and } \quad\left\|\mathbf{B}_{h}^{n+1}\right\|_{\mathbf{L}^{2}(\Omega)} \leq e^{C T}\left\|\mathbf{B}_{h}(0)\right\|_{\mathbf{L}^{2}(\Omega)}
$$

Convergence and error estimates in $L^{2}$. Now we will prove the convergence of the scheme and show some error estimates. First of all we look at the case $\mathbf{B} \in V_{h}^{m}$ and we will change some estimates to adapt the proof when $\mathbf{B} \in W_{h}^{1}$. We set

$$
\mathbf{Y}(t, \mathbf{x})=\mathbf{B}(t, \mathbf{x})+\partial_{t} \mathbf{B}(t, \mathbf{x}) \Delta t
$$

Using Taylor expansion in time we get

$$
\mathbf{B}(t+\Delta t, \mathbf{x})-\mathbf{B}(t, \mathbf{x})-\partial_{t} \mathbf{B}(t, \mathbf{x}) \frac{\Delta t}{2}-\partial_{t} \mathbf{B}(t+\Delta t, \mathbf{x}) \frac{\Delta t}{2}=\mathcal{O}\left(\Delta t^{3}\right) .
$$

Using Taylor expansion and from Equations (2) and (60) we obtain

$$
\begin{aligned}
\partial_{t} \mathbf{B}(t+\Delta t, \mathbf{x})= & -\sum_{i=1}^{2} \partial_{x_{i}}\left(\mathcal{A}_{i}(t+\Delta t, \mathbf{x}) \mathbf{B}(t+\Delta t, \mathbf{x})\right)-\mathcal{C}(t+\Delta t, \mathbf{x}) \mathbf{B}(t+\Delta t, \mathbf{x}) \\
= & -\sum_{i=1}^{2} \partial_{x_{i}}\left(\mathcal{A}_{i}(t+\Delta t, \mathbf{x})\left(\mathbf{B}(t, \mathbf{x})+\partial_{t} \mathbf{B}(t, \mathbf{x}) \Delta t+\mathcal{O}\left(\Delta t^{2}\right)\right)\right) \\
& -\mathcal{C}(t+\Delta t, \mathbf{x})\left(\mathbf{B}(t, \mathbf{x})+\partial_{t} \mathbf{B}(t, \mathbf{x}) \Delta t+\mathcal{O}\left(\Delta t^{2}\right)\right) \\
= & -\sum_{i=1}^{2} \partial_{x_{i}}\left(\mathcal{A}_{i}(t+\Delta t, \mathbf{x}) \mathbf{Y}(t, \mathbf{x})\right)-\mathcal{C}(t+\Delta t, \mathbf{x}) \mathbf{Y}(t, \mathbf{x})+\mathcal{O}\left(\Delta t^{2}\right)
\end{aligned}
$$

From (60) and (61) we get

$$
\mathbf{B}(t+\Delta t, \mathbf{x})=\frac{1}{2} \mathbf{B}(t, \mathbf{x})+\frac{1}{2} \mathbf{Y}(t, \mathbf{x})+\frac{\Delta t}{2} \partial_{t} \mathbf{B}(t+\Delta t, \mathbf{x})+\mathcal{O}\left(\Delta t^{3}\right) .
$$

From (62) and (63) we get

$$
\begin{aligned}
\mathbf{B}(t+\Delta t, \mathbf{x})= & \frac{1}{2} \mathbf{B}(t, \mathbf{x})+\frac{1}{2} \mathbf{Y}(t, \mathbf{x})-\frac{\Delta t}{2} \sum_{i=1}^{2} \partial_{x_{i}}\left(\mathcal{A}_{i}(t+\Delta t, \mathbf{x}) \mathbf{Y}(t, \mathbf{x})\right) \\
& -\frac{\Delta t}{2} \mathcal{C}(t+\Delta t, \mathbf{x}) \mathbf{Y}(t, \mathbf{x})+\mathcal{O}\left(\Delta t^{3}\right)
\end{aligned}
$$

Finally from (60) and (64) we obtain

$$
\begin{aligned}
\mathbf{Y}\left(t^{n}, \mathbf{x}\right) & =\mathbf{B}\left(t^{n}, \mathbf{x}\right)-\Delta t \sum_{i=1}^{2} \partial_{x_{i}}\left(\mathcal{A}_{i}^{n}(\mathbf{x}) \mathbf{B}\left(t^{n}, \mathbf{x}\right)\right)-\Delta t \mathcal{C}^{n}(\mathbf{x}) \mathbf{B}\left(t^{n}, \mathbf{x}\right) \\
\mathbf{B}\left(t^{n+1}, \mathbf{x}\right) & =\frac{1}{2} \mathbf{B}\left(t^{n}, \mathbf{x}\right)+\frac{1}{2} \mathbf{Y}\left(t^{n}, \mathbf{x}\right)-\frac{\Delta t}{2} \sum_{i=1}^{2} \partial_{x_{i}}\left(\mathcal{A}_{i}^{n+1}(\mathbf{x}) \mathbf{Y}\left(t^{n}, \mathbf{x}\right)\right)-\frac{\Delta t}{2} \mathcal{C}\left(t^{n+1}, \mathbf{x}\right) \mathbf{Y}\left(t^{n}, \mathbf{x}\right)+\mathcal{O}\left(\Delta t^{3}\right)
\end{aligned}
$$


If we take the scalar product of (65) and (66) with a test function $\varphi_{h}$, integrate over a cell $K$ and use the Green formula (14) then we get

$$
\begin{aligned}
\int_{K} \mathbf{Y}^{n} \cdot \varphi_{h} \mathrm{~d} \mathbf{x} & =\int_{K} \mathbf{B}^{n} \cdot \boldsymbol{\varphi}_{h} \mathrm{~d} \mathbf{x}+\Delta t \mathcal{F}_{K}^{n}\left(\mathbf{B}^{n}, \boldsymbol{\varphi}_{h}\right) \\
\int_{K} \mathbf{B}^{n+1} \cdot \varphi_{h} \mathrm{~d} \mathbf{x} & =\int_{K}\left(\frac{1}{2} \mathbf{B}^{n}+\frac{1}{2} \mathbf{Y}^{n}\right) \cdot \boldsymbol{\varphi}_{h} \mathrm{~d} \mathbf{x}+\frac{\Delta t}{2} \mathcal{F}_{K}^{n+1}\left(\mathbf{Y}^{n}, \varphi_{h}\right)+\int_{K} \epsilon\left(t^{n}, \mathbf{x}\right) \cdot \boldsymbol{\varphi}_{h} \mathrm{~d} \mathbf{x}
\end{aligned}
$$

where $\left\|\epsilon\left(t^{n}, \mathbf{x}\right)\right\|_{l^{\infty}\left(0, T ; \mathbf{L}^{2}(\Omega)\right)}=\mathcal{O}\left(\Delta t^{3}\right)$. We set the following notations $\delta_{\mathbf{Y}}^{n}=\pi_{h} \mathbf{Y}^{n}-\mathbf{Y}_{h}^{n}, \delta_{\mathbf{B}}^{n}=\pi_{h} \mathbf{B}^{n}-\mathbf{B}_{h}^{n}$, $\mathbf{e}_{\mathbf{Y}}^{n}=\mathbf{Y}^{n}-\mathbf{Y}_{h}^{n}, \mathcal{I}_{\mathbf{Y}}^{n}=\pi_{h} \mathbf{Y}^{n}-\mathbf{Y}^{n}, \mathcal{I}_{\mathbf{B}}^{n}=\pi_{h} \mathbf{B}^{n}-\mathbf{B}^{n}, \mathbf{e}_{\mathbf{B}}^{n}=\mathbf{B}^{n}-\mathbf{B}_{h}^{n}$. If we substract (16) to (67) and (17) to $(68)$ we obtain

$$
\begin{aligned}
\int_{K} \delta_{\mathbf{Y}}^{n} \cdot \varphi_{h} \mathrm{~d} \mathbf{x} & =\int_{K} \delta_{\mathbf{B}}^{n} \cdot \boldsymbol{\varphi}_{h} \mathrm{~d} \mathbf{x}+\mathcal{H}_{K}^{n}\left(\boldsymbol{\varphi}_{h}\right) \\
\int_{K} \delta_{\mathbf{B}}^{n+1} \cdot \varphi_{h} \mathrm{~d} \mathbf{x} & =\int_{K}\left(\frac{1}{2} \delta_{\mathbf{Y}}^{n}+\frac{1}{2} \delta_{\mathbf{B}}^{n}\right) \cdot \boldsymbol{\varphi}_{h} \mathrm{~d} \mathbf{x}+\frac{1}{2} \mathcal{J}_{K}^{n+1}\left(\boldsymbol{\varphi}_{h}\right)
\end{aligned}
$$

where

$$
\mathcal{H}_{K}^{n}\left(\boldsymbol{\varphi}_{h}\right)=\int_{K}\left(\mathcal{I}_{\mathbf{Y}}^{n}-\mathcal{I}_{\mathbf{B}}^{n}\right) \cdot \varphi_{h} \mathrm{~d} \mathbf{x}+\Delta t \mathcal{F}_{K}^{n}\left(\mathbf{B}^{n}, \boldsymbol{\varphi}_{h}\right)-\Delta t \mathcal{F}_{K}^{n}\left(\mathbf{B}_{h}^{n}, \boldsymbol{\varphi}_{h}\right)
$$

and

We set

$$
\mathcal{J}_{K}^{n+1}\left(\boldsymbol{\varphi}_{h}\right)=\int_{K}\left(2 \mathcal{I}_{\mathbf{B}}^{n+1}-\mathcal{I}_{\mathbf{Y}}^{n}-\mathcal{I}_{\mathbf{B}}^{n}+\epsilon\left(t^{n}, \mathbf{x}\right)\right) \cdot \boldsymbol{\varphi}_{h} \mathrm{~d} \mathbf{x}+\Delta t \mathcal{F}_{K}^{n+1}\left(\mathbf{Y}^{n}, \varphi_{h}\right)-\Delta t \mathcal{F}_{K}^{n+1}\left(\mathbf{Y}_{h}^{n}, \varphi_{h}\right) .
$$

$$
\mathcal{H}^{n}=\sum_{K \in \mathcal{T}_{h}} \mathcal{H}_{K}^{n} \mathbb{1}_{K}, \quad \text { and } \quad \mathcal{J}^{n+1}=\sum_{K \in \mathcal{T}_{h}} \mathcal{J}_{K}^{n+1} \mathbb{1}_{K}
$$

If we take $\boldsymbol{\varphi}_{h}=\delta_{\mathrm{B}}^{n}$ in (69) and $\boldsymbol{\varphi}_{h}=\delta_{\mathbf{Y}}^{n}$ in (70), after summing over all the element $K$ of the partition $\mathcal{T}_{h}$ the $\operatorname{sum}(69) / 2+(70)$ gives

$$
\left\|\delta_{\mathbf{B}}^{n+1}\right\|_{\mathbf{L}^{2}(\Omega)}^{2}-\left\|\delta_{\mathbf{B}}^{n}\right\|_{\mathbf{L}^{2}(\Omega)}^{2}-\left\|\delta_{\mathbf{B}}^{n+1}-\delta_{\mathbf{Y}}^{n}\right\|_{\mathbf{L}^{2}(\Omega)}^{2}=\mathcal{H}^{n}\left(\delta_{\mathbf{B}}^{n}\right)+\mathcal{J}^{n+1}\left(\delta_{\mathbf{Y}}^{n}\right) .
$$

In the following we will give estimates for the three terms $\mathcal{H}^{n}\left(\delta_{\mathbf{B}}^{n}\right), \mathcal{J}^{n+1}\left(\delta_{\mathbf{Y}}^{n}\right)$ and $\left\|\delta_{\mathbf{B}}^{n+1}-\delta_{\mathbf{Y}}^{n}\right\|_{\mathbf{L}^{2}(\Omega)}$.

$\underline{\text { Estimate of } \mathcal{H}^{n}\left(\delta_{\mathbf{B}}^{n}\right)}$. From equation (60) and the approximation properties (7) of $\pi_{h}$ we obtain

$$
\left\|\mathcal{I}_{\mathbf{B}}^{n+1}-\mathcal{I}_{\mathbf{B}}^{n}\right\|_{\mathbf{L}^{2}(\Omega)}+\left\|\mathcal{I}_{\mathbf{Y}}^{n}-\mathcal{I}_{\mathbf{B}}^{n}\right\|_{\mathbf{L}^{2}(\Omega)} \leq C \Delta t h^{m+1}\left|\partial_{t} \mathbf{B}\right|_{L^{\infty}\left(0, T ; \mathbf{H}^{m+1}(\Omega)\right)} .
$$

Using (75), the Cauchy-Schwarz and Young inequalities we have for the first term of $\mathcal{H}^{n}\left(\delta_{\mathbf{B}}^{n}\right)$ the estimate

$$
\sum_{K \in \mathcal{T}_{h}} \int_{K}\left(\mathcal{I}_{\mathbf{Y}}^{n}-\mathcal{I}_{\mathbf{B}}^{n}\right) \cdot \delta_{\mathbf{B}}^{n} \mathrm{~d} \mathbf{x} \leq \int_{\Omega}\left\|\mathcal{I}_{\mathbf{Y}}^{n}-\mathcal{I}_{\mathbf{B}}^{n}\right\|_{2}\left\|\delta_{\mathbf{B}}^{n}\right\|_{2} \leq C \Delta t h^{2 m+2}+\Delta t\left\|\delta_{\mathbf{B}}^{n}\right\|_{\mathbf{L}^{2}(\Omega)}^{2}
$$

Now we estimate the term $\Delta t \mathcal{F}^{n}\left(\mathbf{B}^{n}-\pi_{h} \mathbf{B}^{n}, \varphi_{h}\right)$. First we notice the decomposition

$$
\begin{aligned}
\Delta t \sum_{K \in \mathcal{T}_{h}} \int_{K} \mathcal{A}_{i}^{n}\left(\mathbf{B}^{n}-\pi_{h} \mathbf{B}^{n}\right) \cdot \partial_{i} \boldsymbol{\varphi}_{h} \mathrm{~d} \mathbf{x}=\Delta t \sum_{K \in \mathcal{T}_{h}} \int_{K}\left(P_{h} \mathcal{A}_{i}^{n}-\mathcal{A}_{i}^{n}\right)\left(\pi_{h} \mathbf{B}^{n}-\mathbf{B}^{n}\right) \cdot \partial_{i} \boldsymbol{\varphi}_{h} \mathrm{~d} \mathbf{x} \\
-\Delta t \sum_{K \in \mathcal{T}_{h}} \int_{K}\left(\pi_{h} \mathbf{B}^{n}-\mathbf{B}^{n}\right) \cdot P_{h} \mathcal{A}_{i}^{n} \partial_{i} \boldsymbol{\varphi}_{h} \mathrm{~d} \mathbf{x}=\Delta t \sum_{K \in \mathcal{T}_{h}} \int_{K}\left(P_{h} \mathcal{A}_{i}^{n}-\mathcal{A}_{i}^{n}\right)\left(\pi_{h} \mathbf{B}^{n}-\mathbf{B}^{n}\right) \cdot \partial_{i} \boldsymbol{\varphi}_{h} \mathrm{~d} \mathbf{x}
\end{aligned}
$$


because $\mathcal{A}_{i}^{n}$ is symmetric, $P_{h} \mathcal{A}_{i}^{n} \partial_{i} \varphi_{h}=P_{h} u_{i}^{n} \partial_{i} \varphi_{h} \in V_{h}^{m}(\Omega)$ and because of the definition $\pi_{h}$. Now we give an estimate for the term (77). Thanks the approximation properties (7) and (27), the inverse properties (8), the Cauchy-Schwarz and Young inequalities we obtain

$$
\begin{aligned}
& \Delta t \sum_{K \in \mathcal{T}_{h}} \int_{K}\left(P_{h} \mathcal{A}_{i}^{n}-\mathcal{A}_{i}^{n}\right)\left(\pi_{h} \mathbf{B}^{n}-\mathbf{B}^{n}\right) \cdot \partial_{i} \boldsymbol{\varphi}_{h} \mathrm{~d} \mathbf{x} \\
\leq & \sum_{K \in \mathcal{T}_{h}} \int_{K}\left(\Delta t\left\|\mathcal{A}_{i}\right\|_{L^{\infty}\left(0, T ; W^{1, \infty}(\Omega)\right)}^{2}\left\|\pi_{h} \mathbf{B}^{n}-\mathbf{B}^{n}\right\|_{2}^{2}+\Delta t h^{2}\left\|\partial_{i} \boldsymbol{\varphi}_{h}\right\|_{2}^{2}\right) \mathrm{d} \mathbf{x} \leq C h^{2 m+2} \Delta t+\Delta t\left\|\boldsymbol{\varphi}_{h}\right\|_{\mathbf{L}^{2}(\Omega)}^{2}
\end{aligned}
$$

Next we have

$$
\begin{aligned}
\Delta t \sum_{K \in \mathcal{T}_{h}} \int_{K} \mathcal{C}^{n}\left(\pi_{h} \mathbf{B}^{n}-\mathbf{B}^{n}\right) \cdot \boldsymbol{\varphi}_{h} \mathrm{~d} \mathbf{x} & \leq \sum_{K \in \mathcal{T}_{h}} \int_{K}\left(\Delta t\|\mathcal{C}\|_{L^{\infty}\left(0, T ; L^{\infty}(\Omega)\right)}^{2}\left\|\pi_{h} \mathbf{B}^{n}-\mathbf{B}^{n}\right\|_{2}^{2}+\Delta t\left\|\boldsymbol{\varphi}_{h}\right\|_{2}^{2}\right) \mathrm{d} \mathbf{x} \\
& \leq C h^{2 m+2} \Delta t+\Delta t\left\|\boldsymbol{\varphi}_{h}\right\|_{\mathbf{L}^{2}(\Omega)}^{2} .
\end{aligned}
$$

As it has been done for the continuous case (see estimates (31)-(32)) we obtain

$$
\Delta t \sum_{e \in \mathcal{E}_{h}} \int_{e} g^{n}\left(\pi_{h} \mathbf{B}^{n}-\mathbf{B}^{n}\right)_{e} \cdot\left[\boldsymbol{\varphi}_{h}\right]_{e} \mathrm{~d} \Gamma \leq C \Delta t h^{2 m+1}+\frac{\Delta t}{4} \sum_{e \in \mathcal{E}_{h}} \int_{e}\left|\mathcal{A}_{e}^{n}\right|\left[\boldsymbol{\varphi}_{h}\right]_{e} \cdot\left[\boldsymbol{\varphi}_{h}\right]_{e} \mathrm{~d} \Gamma
$$

From (78)-(80) we get

$$
\Delta t \mathcal{F}^{n}\left(\mathbf{B}^{n}-\pi_{h} \mathbf{B}^{n}, \boldsymbol{\varphi}_{h}\right) \leq C \Delta t h^{2 m+1}+3 \Delta t\left\|\boldsymbol{\varphi}_{h}\right\|_{\mathbf{L}^{2}(\Omega)}^{2}+\frac{\Delta t}{4} \sum_{e \in \mathcal{E}_{h}} \int_{e}\left|\mathcal{A}_{e}^{n}\right|\left[\boldsymbol{\varphi}_{h}\right]_{e} \cdot\left[\boldsymbol{\varphi}_{h}\right]_{e} \mathrm{~d} \Gamma
$$

Now we want to estimate the term $\Delta t \mathcal{F}^{n}\left(\mathbf{B}^{n}, \delta_{\mathbf{B}}^{n}\right)-\Delta t \mathcal{F}^{n}\left(\mathbf{B}_{h}^{n}, \delta_{\mathbf{B}}^{n}\right)$. First we notice that

$$
\Delta t \mathcal{F}^{n}\left(\mathbf{B}^{n}, \delta_{\mathbf{B}}^{n}\right)-\Delta t \mathcal{F}^{n}\left(\mathbf{B}_{h}^{n}, \delta_{\mathbf{B}}^{n}\right)=\Delta t \mathcal{F}^{n}\left(\mathbf{B}^{n}-\pi_{h} \mathbf{B}^{n}, \delta_{\mathbf{B}}^{n}\right)+\Delta t \mathcal{F}^{n}\left(\delta_{\mathbf{B}}^{n}, \delta_{\mathbf{B}}^{n}\right)
$$

From (81) we obtain

$$
\Delta t \mathcal{F}^{n}\left(\mathbf{B}^{n}-\pi_{h} \mathbf{B}^{n}, \delta_{\mathbf{B}}^{n}\right) \leq C \Delta t h^{2 m+1}+3 \Delta t\left\|\delta_{\mathbf{B}}^{n}\right\|_{\mathbf{L}^{2}(\Omega)}^{2}+\frac{\Delta t}{4} \sum_{e \in \mathcal{E}_{h}} \int_{e}\left|\mathcal{A}_{e}^{n}\right|\left[\delta_{\mathbf{B}}^{n}\right]_{e} \cdot\left[\delta_{\mathbf{B}}^{n}\right]_{e} \mathrm{~d} \Gamma
$$

From (3) and (44) we get

$$
\Delta t \mathcal{F}^{n}\left(\delta_{\mathbf{B}}^{n}, \delta_{\mathbf{B}}^{n}\right) \leq-\alpha \Delta t\left\|\delta_{\mathbf{B}}^{n}\right\|_{\mathbf{L}^{2}(\Omega)}^{2}-\frac{\Delta t}{2} \sum_{e \in \mathcal{E}_{h}} \int_{e}\left|\mathcal{A}_{e}^{n}\right|\left[\delta_{\mathbf{B}}^{n}\right]_{e} \cdot\left[\delta_{\mathbf{B}}^{n}\right]_{e} \mathrm{~d} \Gamma
$$

From (71), (73), (76), (82)-(84) we get

$$
\mathcal{H}^{n}\left(\delta_{\mathbf{B}}^{n}\right) \leq C \Delta t\left(h^{2 m+1}+h^{2 m+2}\right)+(4-\alpha) \Delta t\left\|\delta_{\mathbf{B}}^{n}\right\|_{\mathbf{L}^{2}(\Omega)}^{2}-\frac{\Delta t}{4} \sum_{e \in \mathcal{E}_{h}} \int_{e}\left|\mathcal{A}_{e}^{n}\right|\left[\delta_{\mathbf{B}}^{n}\right]_{e} \cdot\left[\delta_{\mathbf{B}}^{n}\right]_{e} \mathrm{~d} \Gamma
$$


Estimate of $\mathcal{J}^{n+1}\left(\delta_{\mathbf{Y}}^{n}\right)$. Using (75), the Cauchy-Schwarz and Young inequalities we have for the first term of $\overline{\mathcal{J}^{n+1}\left(\delta_{\mathbf{Y}}^{n}\right) \text { the estimate }}$

$$
\begin{aligned}
& \left.\sum_{K \in \mathcal{T}_{h}} \int_{K}\left(2 \mathcal{I}_{\mathbf{B}}^{n+1}-\mathcal{I}_{\mathbf{Y}}^{n}-\mathcal{I}_{\mathbf{B}}^{n}+\epsilon\left(t^{n} \mathbf{x}\right)\right) \cdot \delta_{\mathbf{Y}}^{n} \mathrm{~d} \mathbf{x} \leq \int_{\Omega} \| 2 \mathcal{I}_{\mathbf{B}}^{n+1}-\mathcal{I}_{\mathbf{Y}}^{n}-\mathcal{I}_{\mathbf{B}}^{n}+\epsilon\left(t^{n} \mathbf{x}\right)\right)\left\|_{2}\right\| \delta_{\mathbf{Y}}^{n} \|_{2} \mathrm{~d} \mathbf{x} \\
& \left.\quad \leq \int_{\Omega}\left(\left\|2\left(\mathcal{I}_{\mathbf{B}}^{n+1}-\mathcal{I}_{\mathbf{B}}^{n}\right)\right\|_{2}+\left\|\mathcal{I}_{\mathbf{B}}^{n}-\mathcal{I}_{\mathbf{Y}}^{n}\right\|_{2}+\| \epsilon\left(t^{n} \mathbf{x}\right)\right) \|_{2}\right)\left\|\delta_{\mathbf{Y}}^{n}\right\|_{2} \mathrm{~d} \mathbf{x} \\
& \quad \leq C(\varepsilon) \Delta t\left(\Delta t^{2}+h^{m+1}\right)^{2}+\varepsilon \Delta t\left\|\delta_{\mathbf{Y}}^{n}\right\|_{\mathbf{L}^{2}(\Omega)}^{2}
\end{aligned}
$$

Now we estimate the term $\Delta t \mathcal{F}^{n+1}\left(\mathbf{Y}^{n}, \delta_{\mathbf{Y}}^{n}\right)-\Delta t \mathcal{F}^{n+1}\left(\mathbf{Y}_{h}^{n}, \delta_{\mathbf{Y}}^{n}\right)$. First we notice that

$$
\Delta t \mathcal{F}^{n+1}\left(\mathbf{Y}^{n}, \delta_{\mathbf{Y}}^{n}\right)-\Delta t \mathcal{F}^{n+1}\left(\mathbf{Y}_{h}^{n}, \delta_{\mathbf{Y}}^{n}\right)=\Delta t \mathcal{F}^{n+1}\left(\mathbf{Y}^{n}-\pi_{h} \mathbf{Y}^{n}, \delta_{\mathbf{Y}}^{n}\right)+\Delta t \mathcal{F}^{n}\left(\delta_{\mathbf{Y}}^{n}, \delta_{\mathbf{Y}}^{n}\right)
$$

The same proof for the estimate (81) leads to

$$
\Delta t \mathcal{F}^{n+1}\left(\mathbf{Y}^{n}-\pi_{h} \mathbf{Y}^{n}, \boldsymbol{\varphi}_{h}\right) \leq C(\varepsilon) \Delta t h^{2 m+1}+\varepsilon \Delta t\left\|\boldsymbol{\varphi}_{h}\right\|_{\mathbf{L}^{2}(\Omega)}^{2}+\frac{\Delta t}{4} \sum_{e \in \mathcal{E}_{h}} \int_{e}\left|\mathcal{A}_{e}^{n}\right|\left[\boldsymbol{\varphi}_{h}\right]_{e} \cdot\left[\boldsymbol{\varphi}_{h}\right]_{e} \mathrm{~d} \Gamma
$$

From (88) we obtain

$$
\Delta t \mathcal{F}^{n}\left(\mathbf{Y}^{n}-\pi_{h} \mathbf{Y}^{n}, \delta_{\mathbf{Y}}^{n}\right) \leq C(\varepsilon) \Delta t h^{2 m+1}+\varepsilon \Delta t\left\|\delta_{\mathbf{Y}}^{n}\right\|_{\mathbf{L}^{2}(\Omega)}^{2}+\frac{\Delta t}{4} \sum_{e \in \mathcal{E}_{h}} \int_{e}\left|\mathcal{A}_{e}^{n}\right|\left[\delta_{\mathbf{Y}}^{n}\right]_{e} \cdot\left[\delta_{\mathbf{Y}}^{n}\right]_{e} \mathrm{~d} \Gamma
$$

From (3) and (44) we get

$$
\Delta t \mathcal{F}^{n}\left(\delta_{\mathbf{Y}}^{n}, \delta_{\mathbf{Y}}^{n}\right) \leq-\alpha \Delta t\left\|\delta_{\mathbf{Y}}^{n}\right\|_{\mathbf{L}^{2}(\Omega)}^{2}-\frac{\Delta t}{2} \sum_{e \in \mathcal{E}_{h}} \int_{e}\left|\mathcal{A}_{e}^{n}\right|\left[\delta_{\mathbf{Y}}^{n}\right]_{e} \cdot\left[\delta_{\mathbf{Y}}^{n}\right]_{e} \mathrm{~d} \Gamma
$$

From (72), (73), (86), (87), (89) and (90) we get

$$
\left.\mathcal{J}^{n+1}\left(\delta_{\mathbf{Y}}^{n}\right) \leq C(\varepsilon) \Delta t\left\{\left(\Delta t^{2}+h^{m+1}\right)^{2}+h^{2 m+1}\right)\right\}+(4 \varepsilon-\alpha) \Delta t\left\|\delta_{\mathbf{Y}}^{n}\right\|_{\mathbf{L}^{2}(\Omega)}^{2}-\frac{\Delta t}{4} \sum_{e \in \mathcal{E}_{h}} \int_{e}\left|\mathcal{A}_{e}^{n}\right|\left[\delta_{\mathbf{Y}}^{n}\right]_{e} \cdot\left[\delta_{\mathbf{Y}}^{n}\right]_{e} \mathrm{~d} \Gamma
$$

Estimate of $\left\|\delta_{\mathbf{B}}^{n+1}-\delta_{\mathbf{Y}}^{n}\right\|_{\mathbf{L}^{2}(\Omega)}$. First we notice that after summing over all elements $K$ of the partition $\mathcal{T}_{h}$ the sum $(70)-(69) / 2$ gives

$$
\int_{\Omega}\left(\delta_{\mathbf{B}}^{n+1}-\delta_{\mathbf{Y}}^{n}\right) \cdot \varphi_{h} \mathrm{~d} \mathbf{x}=\frac{1}{2}\left(\mathcal{J}^{n+1}\left(\boldsymbol{\varphi}_{h}\right)-\mathcal{H}^{n}\left(\boldsymbol{\varphi}_{h}\right)\right)
$$

where

$$
\frac{1}{2}\left(\mathcal{J}^{n+1}\left(\boldsymbol{\varphi}_{h}\right)-\mathcal{H}^{n}\left(\boldsymbol{\varphi}_{h}\right)\right)=\frac{1}{2} \int_{\Omega}\left(2 \mathcal{I}_{\mathbf{B}}^{n+1}-\mathcal{I}_{\mathbf{Y}}^{n}-\mathcal{I}_{\mathbf{B}}^{n}+\epsilon\left(t^{n} \mathbf{x}\right)\right) \cdot \boldsymbol{\varphi}_{h} \mathrm{~d} \mathbf{x}-\frac{1}{2} \int_{\Omega}\left(\mathcal{I}_{\mathbf{Y}}^{n}-\mathcal{I}_{\mathbf{B}}^{n}\right) \cdot \boldsymbol{\varphi}_{h} \mathrm{~d} \mathbf{x}+\mathcal{L}^{n}\left(\boldsymbol{\varphi}_{h}\right)
$$

with $\mathcal{L}^{n}\left(\varphi_{h}\right)=\Delta t\left(\mathcal{F}^{n+1}\left(\mathbf{Y}^{n}, \varphi_{h}\right)-\mathcal{F}^{n+1}\left(\mathbf{Y}_{h}^{n}, \varphi_{h}\right)-\mathcal{F}^{n}\left(\mathbf{B}^{n}, \varphi_{h}\right)+\mathcal{F}^{n}\left(\mathbf{B}_{h}^{n}, \varphi_{h}\right)\right)$. From (75) and (76) we have

$$
\begin{aligned}
\left|\left(\mathcal{I}_{\mathbf{Y}}^{n}-\mathcal{I}_{\mathbf{B}}^{n}, \boldsymbol{\varphi}_{h}\right)_{\Omega}\right| & \leq C \Delta t h^{2 m+2}+\Delta t\left\|\boldsymbol{\varphi}_{h}\right\|_{\mathbf{L}^{2}(\Omega)}^{2} \\
\left|\left(2 \mathcal{I}_{\mathbf{B}}^{n+1}-\mathcal{I}_{\mathbf{Y}}^{n}-\mathcal{I}_{\mathbf{B}}^{n}+\epsilon\left(t^{n} \mathbf{x}\right), \boldsymbol{\varphi}_{h}\right)_{\Omega}\right| & \leq C \Delta t\left(\Delta t^{2}+h^{m+1}\right)^{2}+\Delta t\left\|\boldsymbol{\varphi}_{h}\right\|_{\mathbf{L}^{2}(\Omega)}^{2} .
\end{aligned}
$$


Now let us estimate the term $\mathcal{L}^{n}\left(\varphi_{h}\right)$. First we notice that

$$
\begin{aligned}
\mathcal{L}^{n}\left(\boldsymbol{\varphi}_{h}\right)= & \Delta t\left(\mathcal{F}^{n+1}\left(\pi_{h} \mathbf{Y}^{n}-\mathbf{Y}_{h}^{n}, \boldsymbol{\varphi}_{h}\right)+\mathcal{F}^{n+1}\left(\mathbf{Y}^{n}-\pi_{h} \mathbf{Y}^{n}, \boldsymbol{\varphi}_{h}\right)\right. \\
& \left.+\mathcal{F}^{n}\left(\pi_{h} \mathbf{B}^{n}-\mathbf{B}^{n}, \boldsymbol{\varphi}_{h}\right)+\mathcal{F}^{n}\left(\mathbf{B}_{h}^{n}-\pi_{h} \mathbf{B}^{n}, \boldsymbol{\varphi}_{h}\right)\right) .
\end{aligned}
$$

From (56) and the approximation property (7) of $\pi_{h}$ we get

$$
\begin{aligned}
\left|\Delta t \mathcal{F}^{n}\left(\pi_{h} \mathbf{B}^{n}-\mathbf{B}^{n}, \boldsymbol{\varphi}_{h}\right)\right| & \leq \frac{\varepsilon}{4}\left\|\boldsymbol{\varphi}_{h}\right\|_{\mathbf{L}^{2}(\Omega)}^{2}+C(\varepsilon) \Delta t^{2} h^{2 m}\|\mathbf{B}\|_{L^{\infty}\left(0, T ; \mathbf{H}^{m+1}(\Omega)\right)}^{2}, \\
\left|\Delta t \mathcal{F}^{n+1}\left(\mathbf{Y}^{n}-\pi_{h} \mathbf{Y}^{n}, \boldsymbol{\varphi}_{h}\right)\right| & \leq \frac{\varepsilon}{4}\left\|\boldsymbol{\varphi}_{h}\right\|_{\mathbf{L}^{2}(\Omega)}^{2}+C(\varepsilon) \Delta t^{2} h^{2 m}\|\mathbf{Y}\|_{L^{\infty}\left(0, T ; \mathbf{H}^{m+1}(\Omega)\right)}^{2} .
\end{aligned}
$$

Now let us estimate the remaining term of $\mathcal{L}^{n}\left(\boldsymbol{\varphi}_{h}\right)$, that is to say $\Delta t \mathcal{F}^{n+1}\left(\delta_{\mathbf{Y}}^{n}, \boldsymbol{\varphi}_{h}\right)-\Delta t \mathcal{F}^{n+1}\left(\delta_{\mathbf{B}}^{n}, \boldsymbol{\varphi}_{h}\right)$. First we notice that

$$
\Delta t \mathcal{F}^{n+1}\left(\delta_{\mathbf{Y}}^{n}, \varphi_{h}\right)-\Delta t \mathcal{F}^{n+1}\left(\delta_{\mathbf{B}}^{n}, \varphi_{h}\right)=-\Delta t \mathcal{F}^{n+1}\left(\delta_{\mathbf{B}}^{n}-\delta_{\mathbf{Y}}^{n}, \varphi_{h}\right)+\mathcal{G}\left(\delta_{\mathbf{B}}^{n}, \varphi_{h}\right)
$$

where $\mathcal{G}\left(\cdot, \varphi_{h}\right)$ is defined by (48). From (56) we get

$$
\left|\Delta t \mathcal{F}^{n+1}\left(\delta_{\mathbf{B}}^{n}-\delta_{\mathbf{Y}}^{n}, \boldsymbol{\varphi}_{h}\right)\right| \leq \frac{\varepsilon}{4}\left\|\boldsymbol{\varphi}_{h}\right\|_{\mathbf{L}^{2}(\Omega)}^{2}+C(\varepsilon) \frac{\Delta t^{2}}{h^{2}}\left\|\delta_{\mathbf{B}}^{n}-\delta_{\mathbf{Y}}^{n}\right\|_{\mathbf{L}^{2}(\Omega)}^{2}
$$

and from (52) we get

$$
\left|\mathcal{G}\left(\delta_{\mathbf{B}}^{n}, \boldsymbol{\varphi}_{h}\right)\right| \leq C(\varepsilon)\left(\frac{\Delta t^{4}}{h^{2}}+\Delta t^{4}\right)\left\|\delta_{\mathbf{B}}^{n}\right\|_{\mathbf{L}^{2}(\Omega)}^{2}+\frac{\varepsilon}{4}\left\|\boldsymbol{\varphi}_{h}\right\|_{\mathbf{L}^{2}(\Omega)}^{2}
$$

Now let us estimate the term $\left\|\delta_{\mathbf{B}}^{n}-\delta_{\mathbf{Y}}^{n}\right\|_{\mathbf{L}^{2}(\Omega)}^{2}$ which appears in (100). If we take $\boldsymbol{\varphi}_{h}=\delta_{\mathbf{B}}^{n}-\delta_{\mathbf{Y}}^{n}$ in $(69)$ we get

$$
\left\|\delta_{\mathbf{B}}^{n}-\delta_{\mathbf{Y}}^{n}\right\|_{\mathbf{L}^{2}(\Omega)}^{2}=\mathcal{H}^{n}\left(\delta_{\mathbf{Y}}^{n}-\delta_{\mathbf{B}}^{n}\right)
$$

where

$$
\mathcal{H}^{n}\left(\delta_{\mathbf{Y}}^{n}-\delta_{\mathbf{B}}^{n}\right)=\left(\mathcal{I}_{\mathbf{Y}}^{n}-\mathcal{I}_{\mathbf{B}}^{n}, \delta_{\mathbf{Y}}^{n}-\delta_{\mathbf{B}}^{n}\right)_{\Omega}+\Delta t \mathcal{F}^{n}\left(\mathbf{B}^{n}-\pi_{h} \mathbf{B}^{n}, \delta_{\mathbf{Y}}^{n}-\delta_{\mathbf{B}}^{n}\right)+\Delta t \mathcal{F}^{n}\left(\pi_{h} \mathbf{B}^{n}-\mathbf{B}_{h}^{n}, \delta_{\mathbf{Y}}^{n}-\delta_{\mathbf{B}}^{n}\right)
$$

The estimate of the three terms in the right hand side of (103) gives for $\mathcal{H}^{n}\left(\delta_{\mathbf{Y}}^{n}-\delta_{\mathbf{B}}^{n}\right)$ the inequality

$$
\mathcal{H}^{n}\left(\delta_{\mathbf{Y}}^{n}-\delta_{\mathbf{B}}^{n}\right) \leq \Delta t\left\|\delta_{\mathbf{Y}}^{n}-\delta_{\mathbf{B}}^{n}\right\|_{\mathbf{L}^{2}(\Omega)}^{2}+C(\varepsilon)\left(\Delta t^{2} h^{2 m}+\Delta t h^{2 m+2} \frac{\Delta t^{2}}{h^{2}}\left\|\delta_{\mathbf{B}}^{n}\right\|_{\mathbf{L}^{2}(\Omega)}^{2}\right)+2 \varepsilon\left\|\delta_{\mathbf{Y}}^{n}-\delta_{\mathbf{B}}^{n}\right\|_{\mathbf{L}^{2}(\Omega)}^{2}
$$

If $\varepsilon$ and $\Delta t$ is small enough, (102) and (104) leads to

$$
\left\|\delta_{\mathbf{Y}}^{n}-\delta_{\mathbf{B}}^{n}\right\|_{\mathbf{L}^{2}(\Omega)}^{2} \leq C(\varepsilon)\left(\Delta t^{2} h^{2 m}+\Delta t h^{2 m+2}+\frac{\Delta t^{2}}{h^{2}}\left\|\delta_{\mathbf{B}}^{n}\right\|_{\mathbf{L}^{2}(\Omega)}^{2}\right)
$$

By taking $\varphi_{h}=\delta_{\mathbf{B}}^{n+1}-\delta_{\mathbf{Y}}^{n}$ in (92) and thanks to relation (93)-(101) and (105) we get

$$
\begin{aligned}
\left\|\delta_{\mathbf{B}}^{n+1}-\delta_{\mathbf{Y}}^{n}\right\|_{\mathbf{L}^{2}(\Omega)}^{2} \leq C(\varepsilon) \Delta t\left(h^{2 m+2}\right. & \left.+\left(\Delta t^{2}+h^{m+1}\right)^{2}+\Delta t h^{2 m}+\Delta t^{3} h^{2 m-2}+\Delta t^{2} h^{2 m}\right) \\
& +2(\Delta t+\varepsilon)\left\|\delta_{\mathbf{B}}^{n+1}-\delta_{\mathbf{Y}}^{n}\right\|_{\mathbf{L}^{2}(\Omega)}^{2}+C(\varepsilon)\left(\Delta t^{4}+\frac{\Delta t^{4}}{h^{2}}+\frac{\Delta t^{4}}{h^{4}}\right)\left\|\delta_{\mathbf{B}}^{n}\right\|_{\mathbf{L}^{2}(\Omega)}^{2}
\end{aligned}
$$


If $\Delta t$ and $\varepsilon$ is small enough and $\Delta t \leq C(\varepsilon) h^{4 / 3}$ then (106) leads to

$$
\begin{gathered}
\left\|\delta_{\mathbf{B}}^{n+1}-\delta_{\mathbf{Y}}^{n}\right\|_{\mathbf{L}^{2}(\Omega)}^{2} \leq C(\varepsilon) \Delta t\left(h^{2 m+2}+\left(\Delta t^{2}+h^{m+1}\right)^{2}+h^{2 m+4 / 3}+h^{2 m+8 / 3}\right) \\
+C(\varepsilon)\left(\Delta t^{4}+\Delta t^{5 / 2}+\Delta t\right)\left\|\delta_{\mathbf{B}}^{n}\right\|_{\mathbf{L}^{2}(\Omega)}^{2} \leq C(\varepsilon) \Delta t\left(\left(\Delta t^{2}+h^{m+1}\right)^{2}+h^{2 m+4 / 3}\right)+C(\varepsilon) \Delta t\left\|\delta_{\mathbf{B}}^{n}\right\|_{\mathbf{L}^{2}(\Omega)}^{2} .
\end{gathered}
$$

Finally the estimates (74), (85), (91) and (107) lead to

$$
\begin{gathered}
\left\|\delta_{\mathbf{B}}^{n+1}\right\|_{\mathbf{L}^{2}(\Omega)}^{2} \leq-\frac{\Delta t}{4} \sum_{e \in \mathcal{E}_{h}} \int_{e}\left|\mathcal{A}_{e}^{n}\right|\left[\delta_{\mathbf{B}}^{n}\right]_{e} \cdot\left[\delta_{\mathbf{B}}^{n}\right]_{e} \mathrm{~d} \Gamma-\frac{\Delta t}{4} \sum_{e \in \mathcal{E}_{h}} \int_{e}\left|\mathcal{A}_{e}^{n}\right|\left[\delta_{\mathbf{Y}}^{n}\right]_{e} \cdot\left[\delta_{\mathbf{Y}}^{n}\right]_{e} \mathrm{~d} \Gamma \\
+(1+C(\alpha, \varepsilon) \Delta t)\left\|\delta_{\mathbf{B}}^{n}\right\|_{\mathbf{L}^{2}(\Omega)}^{2}+\Delta t(4 \varepsilon-\alpha)\left\|\delta_{\mathbf{Y}}^{n}\right\|_{\mathbf{L}^{2}(\Omega)}^{2}+C(\varepsilon) \Delta t\left(\left(\Delta t^{2}+h^{m+1}\right)^{2}+h^{2 m+1}+h^{2 m+4 / 3}\right) .
\end{gathered}
$$

Using (105), (108) becomes

$$
\left\|\delta_{\mathbf{B}}^{n+1}\right\|_{\mathbf{L}^{2}(\Omega)}^{2} \leq(1+C(\alpha, \varepsilon) \Delta t)\left\|\delta_{\mathbf{B}}^{n}\right\|_{\mathbf{L}^{2}(\Omega)}^{2}+C(\varepsilon) \Delta t\left(\left(\Delta t^{2}+h^{m+1}\right)^{2}+h^{2 m+1}\right) .
$$

A discrete Gronwall inequality and (109) enable us to obtain

$$
\left\|\delta_{\mathbf{B}}^{n+1}\right\|_{\mathbf{L}^{2}(\Omega)}^{2} \leq\left\|\delta_{\mathbf{B}}^{0}\right\|_{\mathbf{L}^{2}(\Omega)}^{2} e^{C(\alpha, \varepsilon) T}+\left(\Delta t^{2}+h^{m+1}\right)^{2}+h^{2 m+1} \leq C\left(\Delta t^{2}+h^{m+1 / 2}\right)^{2}
$$

because $\left\|\delta_{\mathbf{B}}^{0}\right\|_{\mathbf{L}^{2}(\Omega)}^{2}=\left\|\mathbf{B}^{0}-\pi_{h} \mathbf{B}^{0}\right\|_{\mathbf{L}^{2}(\Omega)}^{2}=\mathcal{O}\left(h^{2 m+2}\right)$. Finally, using (110) we get

$$
\left\|\mathbf{B}_{h}^{n}(\mathbf{x})-\mathbf{B}\left(t^{n}, \mathbf{x}\right)\right\|_{\mathbf{L}^{2}(\Omega)}^{2} \leq\left(\left\|\pi_{h} \mathbf{B}^{n}(\mathbf{x})-\mathbf{B}\left(t^{n}, \mathbf{x}\right)\right\|_{\mathbf{L}^{2}(\Omega)}^{2}+\left\|\delta_{\mathbf{B}}^{n}\right\|_{\mathbf{L}^{2}(\Omega)}^{2}\right)^{1 / 2} \leq C\left(\Delta t^{2}+h^{m+1 / 2}\right) .
$$

To end the proof we notice that (108) implies that

$$
\frac{\Delta t}{4} \sum_{e \in \mathcal{E}_{h}} \int_{e}\left|\mathcal{A}_{e}^{n}\right|\left[\delta_{\mathbf{B}}^{n}\right]_{e} \cdot\left[\delta_{\mathbf{B}}^{n}\right]_{e} \mathrm{~d} \Gamma \leq(1+C(\alpha, \varepsilon) \Delta t)\left\|\delta_{\mathbf{B}}^{n}\right\|_{\mathbf{L}^{2}(\Omega)}^{2}+C \Delta t\left(\Delta t^{2}+h^{m+1 / 2}\right)^{2} .
$$

If we make the sum on the index $n$ from 0 to $N_{T}$ in (111) we obtain

$$
\Delta t \sum_{n=0}^{N_{T}} \sum_{e \in \mathcal{E}_{h}} \int_{e}\left|\mathcal{A}_{e}^{n}\right|\left[\delta_{\mathbf{B}}^{n}\right]_{e} \cdot\left[\delta_{\mathbf{B}}^{n}\right]_{e} \mathrm{~d} \Gamma \leq C\left(\Delta t^{2}+h^{m+1 / 2}\right)^{2} .
$$

Now we suppose that $\mathbf{B}_{h} \in W_{h}^{1}$. Then we see how change the estimates for $\mathcal{H}^{n}\left(\delta_{\mathbf{B}}^{n}\right), \mathcal{J}^{n+1}\left(\delta_{\mathbf{Y}}^{n}\right)$ and $\left\|\delta_{\mathbf{B}}^{n+1}-\delta_{\mathbf{Y}}^{n}\right\|_{\mathbf{L}^{2}(\Omega)}$. First we give a new estimate for the term $\Delta t \mathcal{F}^{n}\left(\mathbf{B}^{n}-\Pi_{h} \mathbf{B}^{n}, \boldsymbol{\varphi}_{h}\right)$. Using the Green formula (14) we obtain

$$
\begin{aligned}
-\Delta t \sum_{i=1}^{2} \int_{\Omega} \mathcal{A}_{i}^{n}\left(\Pi_{h} \mathbf{B}^{n}-\mathbf{B}^{n}\right) \cdot \partial_{i} \varphi_{h} \mathrm{~d} \mathbf{x}+\Delta t \int_{\Omega} \mathcal{C}^{n}\left(\Pi_{h} \mathbf{B}^{n}-\mathbf{B}^{n}\right) \cdot \varphi_{h} \mathrm{~d} \mathbf{x} \\
=\Delta t \sum_{K \in \mathcal{T}_{h}} \int_{K}\left(\sum_{i=1}^{2} \partial_{i}\left(\mathcal{A}_{i}^{n}\left(\Pi_{h} \mathbf{B}^{n}-\mathbf{B}^{n}\right)\right)+\Delta t \mathcal{C}^{n}\left(\Pi_{h} \mathbf{B}^{n}-\mathbf{B}^{n}\right)\right) \cdot \varphi_{h} \mathrm{~d} \mathbf{x} \\
-\Delta t \sum_{K \in \mathcal{T}_{h}} \sum_{e \in \partial K} \int_{e} \mathcal{A}_{e, K}^{n}\left(\Pi_{h} \mathbf{B}^{n}-\mathbf{B}^{n}\right) \cdot \varphi_{h} \mathrm{~d} \Gamma
\end{aligned}
$$


First we look at the term (113). Using the approximation properties (10)-(12), inverse properties (8), the fact that $\operatorname{div} \Pi_{h} \mathbf{B}^{n}=\operatorname{div} \mathbf{B}^{n}=0$ on $K$, the Young and the Cauchy-Schwarz inequalities then we get

$$
\begin{aligned}
& \Delta t \sum_{K \in \mathcal{T}_{h}} \sum_{e \in \partial K} \int_{e} \mathcal{A}_{e, K}^{n}\left(\Pi_{h} \mathbf{B}^{n}-\mathbf{B}^{n}\right) \cdot \boldsymbol{\varphi}_{h} d \Gamma \\
\leq & \sum_{K \in \mathcal{T}_{h}}\left\{2\|\mathbf{u}\|_{L^{\infty}\left(0, T ; L^{\infty}(\Omega)\right)}^{2} \Delta t h^{-1}\left\|\Pi_{h} \mathbf{B}^{n}-\mathbf{B}^{n}\right\|_{\mathbf{L}^{2}(\partial K)}^{2}+\Delta t h\left\|\boldsymbol{\varphi}_{h}\right\|_{\mathbf{L}^{2}(\partial K)}^{2}\right\} \\
\leq & \sum_{K \in \mathcal{T}_{h}}\left\{2\|\mathbf{u}\|_{L^{\infty}\left(0, T ; L^{\infty}(\Omega)\right)}^{2} \Delta t h^{-1}\left\|\Pi_{h} \mathbf{B}^{n}-\mathbf{B}^{n}\right\|_{\mathcal{W}(K)}^{2}+\Delta t\left\|\boldsymbol{\varphi}_{h}\right\|_{\mathbf{L}^{2}(K)}^{2}\right\} \\
\leq & \sum_{K \in \mathcal{T}_{h}}\left\{2\|\mathbf{u}\|_{L^{\infty}\left(0, T ; L^{\infty}(\Omega)\right)}^{2} \Delta t h^{-1}\left(\left\|\Pi_{h} \mathbf{B}^{n}-\mathbf{B}^{n}\right\|_{\mathbf{H}(\operatorname{curl} ; K)}^{2}+\left\|\left(\Pi_{h} \mathbf{B}^{n}-\mathbf{B}^{n}\right) \times \boldsymbol{\nu}\right\|_{L^{2}(\partial K)}^{2}\right)+\Delta t\left\|\boldsymbol{\varphi}_{h}\right\|_{\mathbf{L}^{2}(K)}^{2}\right\} \\
\leq & C\left(T,\|\mathbf{u}\|_{L^{\infty}\left(0, T ; L^{\infty}(\Omega)\right)},\|\mathbf{B}\|_{L^{\infty}\left(0, T ; \mathbf{H}^{2}(\Omega)\right)}\right) \Delta t h+\Delta t\left\|\boldsymbol{\varphi}_{h}\right\|_{\mathbf{L}^{2}(\Omega)}^{2} .
\end{aligned}
$$

As $\operatorname{div} \Pi_{h} \mathbf{B}^{n}=\operatorname{div} \mathbf{B}^{n}=0$ on $K$ the term (112) can be recasted in

$$
\begin{aligned}
& \Delta t \sum_{K \in \mathcal{T}_{h}} \int_{K}\left(\sum_{i=1}^{2} \partial_{i}\left(\mathcal{A}_{i}^{n}\left(\Pi_{h} \mathbf{B}^{n}-\mathbf{B}^{n}\right)\right)+\mathcal{C}^{n}\left(\Pi_{h} \mathbf{B}^{n}-\mathbf{B}^{n}\right)\right) \cdot \boldsymbol{\varphi}_{h} \mathrm{~d} \mathbf{x} \\
& =\Delta t \sum_{K \in \mathcal{T}_{h}} \int_{K}\left\{\left(\left(\Pi_{h} \mathbf{B}^{n}-\mathbf{B}^{n}\right) \cdot \nabla\right) \mathbf{u}^{n}+\left(\Pi_{h} \mathbf{B}^{n}-\mathbf{B}^{n}\right) \operatorname{div} \mathbf{u}^{n}+\left(\mathbf{u}^{n} \cdot \nabla\right)\left(\Pi_{h} \mathbf{B}^{n}-\mathbf{B}^{n}\right)\right\} \cdot \boldsymbol{\varphi}_{h} \mathrm{~d} \mathbf{x} .
\end{aligned}
$$

Now we have to estimate the three terms of (115). For the two first term of (115) we get

$$
\begin{aligned}
& \Delta t \sum_{K \in \mathcal{T}_{h}} \int_{K}\left(\left(\Pi_{h} \mathbf{B}^{n}-\mathbf{B}^{n}\right) \cdot \nabla\right) \mathbf{u}^{n} \cdot \boldsymbol{\varphi}_{h} \mathrm{~d} \mathbf{x} \\
\leq & \sum_{K \in \mathcal{T}_{h}}\left\{4\|\mathbf{u}\|_{L^{\infty}\left(0, T ; W^{1, \infty}(\Omega)\right)}^{2} \Delta t\left\|\Pi_{h} \mathbf{B}^{n}-\mathbf{B}^{n}\right\|_{\mathbf{L}^{2}(K)}^{2}+\Delta t\left\|\boldsymbol{\varphi}_{h}\right\|_{\mathbf{L}^{2}(K)}^{2}\right\} \\
\leq & C\left(T,\|\mathbf{u}\|_{L^{\infty}\left(0, T ; W^{1, \infty}(\Omega)\right)},|\mathbf{B}|_{L^{\infty}\left(0, T ; \mathbf{H}^{1}(\Omega)\right)}\right) h^{2}+\Delta t\left\|\boldsymbol{\varphi}_{h}\right\|_{\mathbf{L}^{2}(\Omega)}^{2},
\end{aligned}
$$

and

$$
\begin{aligned}
& \Delta t \sum_{K \in \mathcal{T}_{h}} \int_{K}\left(\Pi_{h} \mathbf{B}^{n}-\mathbf{B}^{n}\right) \operatorname{div} \mathbf{u}^{n} \cdot \boldsymbol{\varphi}_{h} \mathrm{~d} \mathbf{x} \\
\leq & \sum_{K \in \mathcal{T}_{h}}\left\{\|\operatorname{div} \mathbf{u}\|_{L^{\infty}\left(0, T ; L^{\infty}(\Omega)\right)}^{2} \Delta t\left\|\Pi_{h} \mathbf{B}^{n}-\mathbf{B}^{n}\right\|_{\mathbf{L}^{2}(K)}^{2}+\Delta t\left\|\boldsymbol{\varphi}_{h}\right\|_{\mathbf{L}^{2}(K)}^{2}\right\} \\
\leq & C\left(T,\|\operatorname{div} \mathbf{u}\|_{L^{\infty}\left(0, T ; L^{\infty}(\Omega)\right)},|\mathbf{B}|_{L^{\infty}\left(0, T ; \mathbf{H}^{1}(\Omega)\right)}\right) h^{2}+\Delta t\left\|\boldsymbol{\varphi}_{h}\right\|_{\mathbf{L}^{2}(\Omega)}^{2} .
\end{aligned}
$$


Using the approximation properties (10)-(12), the continuous imbedding (13), the fact that $\operatorname{div} \Pi_{h} \mathbf{B}=\operatorname{div} \mathbf{B}=0$ on $K$ and the Cauchy-Schwarz inequality then we get

$$
\begin{aligned}
& \Delta t \sum_{K \in \mathcal{T}_{h}} \int_{K}\left(\mathbf{u}^{n} \cdot \nabla\right)\left(\Pi_{h} \mathbf{B}^{n}-\mathbf{B}^{n}\right) \cdot \boldsymbol{\varphi}_{h} \mathrm{~d} \mathbf{x} \mathrm{d} t \\
\leq & \sum_{K \in \mathcal{T}_{h}}\left\{4\|\mathbf{u}\|_{L^{\infty}\left(0, T ; L^{\infty}(\Omega)\right)}^{2} \Delta t\left\|\Pi_{h} \mathbf{B}^{n}-\mathbf{B}^{n}\right\|_{\mathbf{H}^{1}(K)}^{2}+\Delta t\left\|\boldsymbol{\varphi}_{h}\right\|_{\mathbf{L}^{2}(K)}^{2}\right\} \\
\leq & \sum_{K \in \mathcal{T}_{h}}\left\{C \Delta t\left(\left\|\Pi_{h} \mathbf{B}^{n}-\mathbf{B}^{n}\right\|_{\mathbf{H}_{(\operatorname{curl} ; K)}}^{2}+\sum_{e \in \partial K}\left\|\left(\Pi_{h} \mathbf{B}^{n}-\mathbf{B}^{n}\right) \times \boldsymbol{\nu}_{e}\right\|_{\mathbf{H}^{1 / 2}(e)}^{2}\right)+\Delta t\left\|\boldsymbol{\varphi}_{h}\right\|_{\mathbf{L}^{2}(K)}^{2}\right\} \\
\leq & C\left(T,\|\mathbf{u}\|_{L^{\infty}\left(0, T ; L^{\infty}(\Omega)\right)},\|\mathbf{B}\|_{L^{\infty}\left(0, T ; \mathbf{H}^{2}(\Omega)\right)}\right) h \Delta t+\Delta t\left\|\boldsymbol{\varphi}_{h}\right\|_{\mathbf{L}^{2}(\Omega)}^{2} .
\end{aligned}
$$

Using (112)-(118) and looking what it has been done in the case of the continuous case for the estimates (31) and (35) we obtain

$$
\Delta t \mathcal{F}^{n}\left(\mathbf{B}^{n}-\Pi_{h} \mathbf{B}^{n}, \boldsymbol{\varphi}_{h}\right) \leq C \Delta t h+3 \Delta t\left\|\boldsymbol{\varphi}_{h}\right\|_{\mathbf{L}^{2}(\Omega)}^{2}+\frac{\Delta t}{4} \sum_{e \in \mathcal{E}_{h}} \int_{e}\left|\mathcal{A}_{e}^{n}\right|\left[\boldsymbol{\varphi}_{h}\right]_{e} \cdot\left[\boldsymbol{\varphi}_{h}\right]_{e} \mathrm{~d} \Gamma
$$

By changing the approximation properties of $\pi_{h}$ by those of $\Pi_{h}$, using (119), and following what it has been done in the case where $\mathbf{B} \in V_{h}^{m}$, the new estimates for $\mathcal{H}^{n}\left(\delta_{\mathbf{B}}^{n}\right), \mathcal{J}^{n+1}\left(\delta_{\mathbf{Y}}^{n}\right)$ and $\left\|\delta_{\mathbf{B}}^{n+1}-\delta_{\mathbf{Y}}^{n}\right\|_{\mathbf{L}^{2}(\Omega)}$ are

$$
\begin{gathered}
\mathcal{H}^{n}\left(\delta_{\mathbf{B}}^{n}\right) \leq C \Delta t\left(h+h^{2}\right)+(4-\alpha) \Delta t\left\|\delta_{\mathbf{B}}^{n}\right\|_{\mathbf{L}^{2}(\Omega)}^{2}-\frac{\Delta t}{4} \sum_{e \in \mathcal{E}_{h}} \int_{e}\left|\mathcal{A}_{e}^{n}\right|\left[\delta_{\mathbf{B}}^{n}\right]_{e} \cdot\left[\delta_{\mathbf{B}}^{n}\right]_{e} \mathrm{~d} \Gamma, \\
\left.\mathcal{J}^{n+1}\left(\delta_{\mathbf{Y}}^{n}\right) \leq C(\varepsilon) \Delta t\left\{\left(\Delta t^{2}+h\right)^{2}+h\right)\right\}+(4 \varepsilon-\alpha) \Delta t\left\|\delta_{\mathbf{Y}}^{n}\right\|_{\mathbf{L}^{2}(\Omega)}^{2}-\frac{\Delta t}{4} \sum_{e \in \mathcal{E}_{h}} \int_{e}\left|\mathcal{A}_{e}^{n}\right|\left[\delta_{\mathbf{Y}}^{n}\right]_{e} \cdot\left[\delta_{\mathbf{Y}}^{n}\right]_{e} \mathrm{~d} \Gamma
\end{gathered}
$$

and

$$
\left\|\delta_{\mathbf{B}}^{n+1}-\delta_{\mathbf{Y}}^{n}\right\|_{\mathbf{L}^{2}(\Omega)}^{2} \leq C(\varepsilon) \Delta t\left(\left(\Delta t^{2}+h\right)^{2}+h^{4 / 3}\right)+C(\varepsilon) \Delta t\left\|\delta_{\mathbf{B}}^{n}\right\|_{\mathbf{L}^{2}(\Omega)}^{2} .
$$

Therefore (120)-(122) and (74) leads to

$$
\left\|\mathbf{B}_{h}^{n}(\mathbf{x})-\mathbf{B}\left(t^{n}, \mathbf{x}\right)\right\|_{\mathbf{L}^{2}(\Omega)}+\left(\Delta t \sum_{n=0}^{N_{T}} \sum_{e \in \mathcal{E}_{h}} \int_{e}\left|\mathcal{A}_{e}^{n}\right|\left[\delta_{\mathbf{B}}^{n}\right]_{e} \cdot\left[\delta_{\mathbf{B}}^{n}\right]_{e} \mathrm{~d} \Gamma\right)^{1 / 2} \leq C\left(\Delta t^{2}+h^{1 / 2}\right) .
$$

\section{Conclusion}

Here we have proven the convergence of some locally divergence-free discontinuous Galerkin methods for the induction equation. The next step is to see how these schemes behave numerically and compare them with other known schemes. For this purpose, we can couple the discontinuous Galerkin scheme for the induction equation with a finite volume scheme for the hydrodynamic equations or use a discontinuous Galerkin scheme for both where the hydrodynamic variables can be approximated in a different finite dimensional space. This work will be the matter of a future collaboration. 


\section{Appendix A. Proof of theorem 2}

Thanks to the theorem 1 there exists $\chi \in V_{h}^{m}(\Omega)$ such that

$$
\|\mathbf{v}-\chi\|_{\mathbf{L}^{2}(K)} \leq C h_{K}^{m+1}|\mathbf{v}|_{\mathbf{H}^{m+1}(K)}, \quad \forall K \in \mathcal{T}_{h}
$$

Moreover we have

$$
\left\|\pi_{h} \mathbf{v}-\mathbf{v}\right\|_{\mathbf{L}^{2}(\Omega)}^{2} \leq \sum_{K \in \mathcal{T}_{h}}\left\|\pi_{h} \mathbf{v}-\mathbf{v}\right\|_{\mathbf{L}^{2}(K)}^{2} \leq \sum_{K \in \mathcal{T}_{h}}\left(\left\|\pi_{K}(\mathbf{v}-\boldsymbol{\chi})\right\|_{\mathbf{L}^{2}(K)}^{2}+\left\|\pi_{K} \boldsymbol{\chi}-\boldsymbol{\chi}\right\|_{\mathbf{L}^{2}(K)}^{2}+\|\chi-\mathbf{v}\|_{\mathbf{L}^{2}(K)}^{2}\right)
$$

From (123) we have $\|\mathbf{v}-\boldsymbol{\chi}\|_{\mathbf{L}^{2}(K)}^{2} \leq C h_{K}^{2 m+2}|\mathbf{v}|_{\mathbf{H}^{m+1}(K)}^{2}$. From (6), (123) and Cauchy-Schwarz inequality we obtain that $\left\|\pi_{K}(\mathbf{v}-\boldsymbol{\chi})\right\|_{\mathbf{L}^{2}(K)}^{2} \leq\left\|\pi_{K}\right\|\|\mathbf{v}-\boldsymbol{\chi}\|_{\mathbf{L}^{2}(K)}^{2} \leq\|\mathbf{v}-\boldsymbol{\chi}\|_{\mathbf{L}^{2}(K)}^{2} \leq C h_{K}^{2 m+2}|\mathbf{v}|_{\mathbf{H}^{m+1}(K)}^{2}$. Using the fact that $\chi$ satisfies $(6)$, if we choose $\phi=\left(\pi_{K} \boldsymbol{\chi}-\boldsymbol{\chi}\right)_{\left.\right|_{K}}$, then we have $\left\|\pi_{K} \boldsymbol{\chi}-\boldsymbol{\chi}\right\|_{\mathbf{L}^{2}(K)}^{2}=0$. Then we obtain

$$
\left\|\pi_{h} \mathbf{v}-\mathbf{v}\right\|_{\mathbf{L}^{2}(\Omega)}^{2} \leq \sum_{K \in \mathcal{T}_{h}}\left\|\pi_{h} \mathbf{v}-\mathbf{v}\right\|_{\mathbf{L}^{2}(K)}^{2} \leq C \sum_{K \in \mathcal{T}_{h}} h_{K}^{2 m+2}|\mathbf{v}|_{\mathbf{H}^{m+1}(K)}^{2} \leq C \sigma^{2} h^{2 m+2}|\mathbf{v}|_{\mathbf{H}^{m+1}(\Omega)}^{2}
$$

The proof of the inverse properties (8) which are due to the assumptions of the partition $\mathcal{T}_{h}$ (see [2]) can be found in [5]. Following the proof of the approximation estimate (7), using (5) and the first inverse inequality of (8) we get $\left\|\pi_{h} \mathbf{v}-\mathbf{v}\right\|_{\mathbf{H}^{k}(K)} \leq C h^{m+1-k}|\mathbf{v}|_{\mathbf{H}^{m+1}(K)}, \forall K \in \mathcal{T}_{h}, \mathbf{v} \in \mathbf{S}^{m+1}(\Omega)$ with $k \leq m+1$. From this last approximation result and the interpolation theorem of Lions and Petree [18] or theorem 1.4 in [15] we obtain approximation result (9).

\section{Appendix B. Proof of the Stability Result of theorem 4}

Here we prove the $L^{2}$-stability of the semi-discretized scheme in space. This proof remains true for the two choices of approximation space. Let $\mathcal{L}_{h}(\cdot, \cdot)$ the bilinear form defined by

$$
\begin{aligned}
\mathcal{L}_{h}\left(\mathbf{B}_{h}, \boldsymbol{\varphi}_{h}\right)=\int_{0}^{T} \int_{\Omega} \partial_{t} \mathbf{B}_{h} \cdot \boldsymbol{\varphi}_{h} \mathrm{~d} \mathbf{x} \mathrm{d} t-\sum_{i=1}^{2} \int_{0}^{T} \sum_{K \in \mathcal{T}_{h}} \int_{K} \mathcal{A}_{i} \mathbf{B}_{h} \cdot \partial_{i} \boldsymbol{\varphi}_{h} \mathrm{~d} \mathbf{x} \mathrm{d} t \\
\quad+\int_{0}^{T} \sum_{K \in \mathcal{T}_{h}} \sum_{e \in \partial K} \int_{e} g\left(\mathbf{B}_{h}\right)_{e} \cdot \boldsymbol{\varphi}_{h} \mathrm{~d} \Gamma \mathrm{d} t+\int_{0}^{T} \int_{\Omega} \mathcal{C} \mathbf{B}_{h} \cdot \boldsymbol{\varphi}_{h} \mathrm{~d} \mathbf{x} \mathrm{d} t
\end{aligned}
$$

with $\boldsymbol{\varphi}_{h} \in V_{h}^{m}$ or $\boldsymbol{\varphi}_{h} \in W_{h}^{1}$. If we set $\left[\boldsymbol{\varphi}_{h}\right]_{e}=\left(\boldsymbol{\varphi}_{K_{e}}-\boldsymbol{\varphi}_{K}\right)_{\left.\right|_{e}}$ then we can rewritten (124) as

$$
\begin{aligned}
\mathcal{L}_{h}\left(\mathbf{B}_{h}, \boldsymbol{\varphi}_{h}\right)=\int_{0}^{T} \int_{\Omega} \partial_{t} \mathbf{B}_{h} \cdot \boldsymbol{\varphi}_{h} \mathrm{~d} \mathbf{x} \mathrm{d} t-\sum_{i=1}^{2} \int_{0}^{T} \int_{\Omega} \mathcal{A}_{i} \mathbf{B}_{h} \cdot \partial_{i} \boldsymbol{\varphi}_{h} \mathrm{~d} \mathbf{x} \mathrm{d} t \\
\quad-\int_{0}^{T} \sum_{e \in \mathcal{E}_{h}} \int_{e} g\left(\mathbf{B}_{h}\right)_{e} \cdot\left[\boldsymbol{\varphi}_{h}\right]_{e} \mathrm{~d} \Gamma \mathrm{d} t+\int_{0}^{T} \int_{\Omega} \mathcal{C} \mathbf{B}_{h} \cdot \boldsymbol{\varphi}_{h} \mathrm{~d} \mathbf{x} \mathrm{d} t
\end{aligned}
$$


where $\mathcal{E}_{h}$ denotes the set of all the faces $e$ of the partition $\mathcal{T}_{h}$.

If we take $\varphi_{h}=\mathbf{B}_{h}$ in (125) we obtain

$$
\begin{aligned}
\mathcal{L}_{h}\left(\mathbf{B}_{h}, \varphi_{h}\right)= & \frac{1}{2}\|\mathbf{B}(T)\|_{\mathbf{L}^{2}(\Omega)}^{2}-\frac{1}{2}\|\mathbf{B}(0)\|_{\mathbf{L}^{2}(\Omega)}^{2}+\int_{0}^{T} \int_{\Omega} \mathcal{C} \mathbf{B}_{h} \cdot \mathbf{B}_{h} \mathrm{~d} \mathbf{x} \mathrm{d} t \\
& -\sum_{K \in \mathcal{T}_{h}} \sum_{i=1}^{2} \int_{0}^{T} \int_{K} \mathcal{A}_{i} \mathbf{B}_{K} \cdot \partial_{i} \mathbf{B}_{K} \mathrm{~d} \mathbf{x} \mathrm{d} t \\
& -\int_{0}^{T} \sum_{e \in \mathcal{E}_{h}} \int_{e} g\left(\mathbf{B}_{h}\right)_{e} \cdot\left[\mathbf{B}_{h}\right]_{e} \mathrm{~d} \Gamma \mathrm{d} t .
\end{aligned}
$$

From the definition (18) of the upwind flux, the term (127) can be rewritten as follows:

$$
\int_{0}^{T} \sum_{e \in \mathcal{E}_{h}} \int_{e} g\left(\mathbf{B}_{h}\right)_{e} \cdot\left[\mathbf{B}_{h}\right]_{e} \mathrm{~d} \Gamma \mathrm{d} t=\int_{0}^{T} \sum_{e \in \mathcal{E}_{h}} \int_{e} \mathcal{A}_{e, K} \overline{\mathbf{B}}_{h, e} \cdot\left[\mathbf{B}_{h}\right]_{e} \mathrm{~d} \Gamma \mathrm{d} t-\frac{1}{2} \int_{0}^{T} \sum_{e \in \mathcal{E}_{h}} \int_{e}\left|\mathcal{A}_{e, K}\right|\left[\mathbf{B}_{h}\right]_{e} \cdot\left[\mathbf{B}_{h}\right]_{e} \mathrm{~d} \Gamma \mathrm{d} t
$$

where $\overline{\mathbf{B}}_{h, e}=\left(\frac{\mathbf{B}_{K}+\mathbf{B}_{K_{e}}}{2}\right)_{\left.\right|_{e}}$. Now let us rewrite the term (126). By using the fact that

$$
\mathcal{A}_{i} \mathbf{B}_{K} \cdot \partial_{i} \mathbf{B}_{K}=\frac{1}{2} \partial_{i}\left(\mathcal{A}_{i} \mathbf{B}_{K} \cdot \mathbf{B}_{K}\right)-\frac{1}{2} \partial_{i} \mathcal{A}_{i} \mathbf{B}_{K} \cdot \mathbf{B}_{K}
$$

and the Green formula (14), we obtain:

$$
\begin{aligned}
& -\sum_{K \in \mathcal{T}_{h}} \sum_{i=1}^{2} \int_{0}^{T} \int_{K} \mathcal{A}_{i} \mathbf{B}_{K} \cdot \partial_{i} \mathbf{B}_{K} \mathrm{~d} \mathbf{x} \mathrm{d} t \\
& \quad=\frac{1}{2} \int_{0}^{T} \int_{\Omega} \nabla \cdot \mathbf{u}\left\|\mathbf{B}_{h}\right\|_{2}^{2} \mathrm{~d} \mathbf{x} \mathrm{d} t-\frac{1}{2} \int_{0}^{T} \sum_{K \in \mathcal{T}_{h}} \sum_{e \in \partial K} \sum_{i=1}^{2} \int_{e} \mathcal{A}_{i} \mathbf{B}_{K} \cdot \mathbf{B}_{K} \nu_{e, K}^{i} \mathrm{~d} \Gamma \mathrm{d} t .
\end{aligned}
$$

From the fact that $\mathcal{A}_{i}$ is symmetric and hence $\mathcal{A}_{e, K}$ is, and the relation $\mathcal{A}_{e, K_{e}}=-\mathcal{A}_{e, K}$, the second term of (130) can rewritten as

$$
\begin{aligned}
-\frac{1}{2} & \int_{0}^{T} \sum_{K \in \mathcal{T}_{h}} \sum_{e \in \partial K} \sum_{i=1}^{2} \int_{e} \mathcal{A}_{i} \mathbf{B}_{K} \cdot \mathbf{B}_{K} \nu_{e, K}^{i} \mathrm{~d} \Gamma \mathrm{d} t=-\frac{1}{2} \int_{0}^{T} \sum_{K \in \mathcal{T}_{h}} \sum_{e \in \partial K} \int_{e} \mathcal{A}_{e, K} \mathbf{B}_{K} \cdot \mathbf{B}_{K} \mathrm{~d} \Gamma \mathrm{d} t \\
& =-\frac{1}{2} \int_{0}^{T} \sum_{e \in \mathcal{E}_{h}} \int_{e}\left(\mathcal{A}_{e, K} \mathbf{B}_{K} \cdot \mathbf{B}_{K}-\mathcal{A}_{e, K} \mathbf{B}_{K_{e}} \cdot \mathbf{B}_{K_{e}}\right) \mathrm{d} \Gamma \mathrm{d} t \\
& =\frac{1}{2} \int_{0}^{T} \sum_{e \in \mathcal{E}_{h}} \int_{e}\left(\mathbf{B}_{K}^{T}+\mathbf{B}_{K_{e}}^{T}\right) \mathcal{A}_{e, K}\left(\mathbf{B}_{K_{e}}-\mathbf{B}_{K}\right) \mathrm{d} \Gamma \mathrm{d} t \\
& =\int_{0}^{T} \sum_{e \in \mathcal{E}_{h}} \int_{e} \overline{\mathbf{B}}_{h, e}^{T} \mathcal{A}_{e, K}\left[\mathbf{B}_{h}\right]_{e} \mathrm{~d} \Gamma \mathrm{d} t=\int_{0}^{T} \sum_{e \in \mathcal{E}_{h}} \int_{e} \mathcal{A}_{e, K} \overline{\mathbf{B}}_{h, e} \cdot\left[\mathbf{B}_{h}\right]_{e} \mathrm{~d} \Gamma \mathrm{d} t
\end{aligned}
$$


From (128)-(131) we can write

$$
\begin{aligned}
\mathcal{L}_{h}\left(\mathbf{B}_{h}, \mathbf{B}_{h}\right) & =\frac{1}{2}\left\|\mathbf{B}_{h}(T)\right\|_{\mathbf{L}^{2}(\Omega)}^{2}-\frac{1}{2}\left\|\mathbf{B}_{h}(0)\right\|_{\mathbf{L}^{2}(\Omega)}^{2}+\frac{1}{2} \int_{0}^{T} \sum_{e \in \mathcal{E}_{h}} \int_{e}\left|\mathcal{A}_{e, K}\right|\left[\mathbf{B}_{h}\right]_{e} \cdot\left[\mathbf{B}_{h}\right]_{e} \mathrm{~d} \Gamma \mathrm{d} t \\
& +\int_{0}^{T} \int_{\Omega} \mathcal{C} \mathbf{B}_{h} \cdot \mathbf{B}_{h} \mathrm{~d} \mathbf{x} \mathrm{d} t+\frac{1}{2} \int_{0}^{T} \int_{\Omega} \nabla \cdot \mathbf{u}\left\|\mathbf{B}_{h}\right\|_{2}^{2} \mathrm{~d} \mathbf{x} \mathrm{d} t .
\end{aligned}
$$

Since $\mathcal{L}_{h}\left(\mathbf{B}_{h}, \mathbf{B}_{h}\right)=0$ then from the previous equation we get the inequality

$$
\left\|\mathbf{B}_{h}(t)\right\|_{\mathbf{L}^{2}(\Omega)}^{2} \leq\left\|\mathbf{B}_{h}(0)\right\|_{\mathbf{L}^{2}(\Omega)}^{2}+\left(2\|\mathcal{C}\|_{L^{\infty}([0, T] \times \Omega)}+\|\nabla \cdot \mathbf{u}\|_{L^{\infty}([0, T] \times \Omega)}\right) \int_{0}^{t}\left\|\mathbf{B}_{h}(s)\right\|_{\mathbf{L}^{2}(\Omega)}^{2} ; \mathrm{d} s
$$

for all $t \leq T$. Then using a Gronwall inequality we obtain

$$
\left\|\mathbf{B}_{h}(t)\right\|_{\mathbf{L}^{2}(\Omega)} \leq\left\|\mathbf{B}_{h}(0)\right\|_{\mathbf{L}^{2}(\Omega)} e^{\left(\|\mathcal{C}\|_{L^{\infty}([0, T] \times \Omega)}+\frac{1}{2}\|\nabla \cdot \mathbf{u}\|_{L^{\infty}([0, T] \times \Omega)}\right) t}, \quad \forall t \leq T .
$$

\section{REFERENCES}

[1] S. Alinhac and P. Gérard, Opérateurs pseudo-différentiels et théorème de Nash-Moser, CNRS Éditions (1991).

[2] G.A. Baker, W.N. Jureidini and O.A. Karakashian, A piecewise solenoidal vector fields and the stokes problem. SIAM J. Numer. Anal. 27 (1990) 1466-1485.

[3] D.S. Balsara and D.S. Spicer, A piecewise solenoidal vector fields and the stokes problem. J. Comput. Phys. 149 (1999) 270-292.

[4] J.U. Brackbill and D.C. Barnes. The effect of nonzero $\nabla \cdot \mathbf{B}$ on the numerical solution of the magnetohydrodynamic equations. J. Comput. Phys. 35 (1980) 426.

[5] P.G. Ciarlet, Basic error estimates for elliptic problems, in Handbook of numerical analysis, P.G. Ciarlet and J.-L. Lions, Eds., North-Holland (1991) 17-351.

[6] B. Cockburn, Discontinuous Galerkin methods for convection dominated problems, in High-order methods for computational physics, Springer, Berlin. Lect. Notes Comput. Sci. Eng. 9 (1999) 69-224.

[7] B. Cockburn, F. Li and C.-W. Shu, Locally divergence-free discontinuous Galerkin-methods for the Maxwell equations. J. Comput. Phys. 194 (2004) 588-610.

[8] M. Costabel, A remark on the regularity of solutions of Maxwell's equations on Lipschitz domains. Math. Method Appl. Sci. 12 (1990) 365-368.

[9] M. Costabel and M. Dauge, Un résultat de densité pour les équations de Maxwell régularisées dans un domaine lipschitzien. C. R. Acad. Sci. Paris Sér. I 327 849-854 (1998).

[10] W. Dai and P.R. Woodward, On the divergence-free condition and conservation laws in numerical simulations for supersonic magnetohydrodynamic flow. Astrophys. J. 494 (1998) 317.

[11] A. Dedner, F. Kemm, D. Kröner, C.-D. Munz, T. Schnitzer and M. Wesenberg, Hyperbolic Divergence cleaning for the MHD equations. J. Comput. Phys. 175 (2002) 645-673.

[12] C.R. Evans and J.F. Hawley, Simulation of magnetohydrodynamic flows, a constrained transport method. Astrophys. J. 332 (1988) 659

[13] C. Foias and R. Temam, Remarques sur les équations de Navier-Stokes et les phénoménes successifs de bifurcation. Ann. Sci. Norm. Sup. Pisa Sér. IV 5 (1978) 29-63.

[14] K.O. Friedrichs, Symmetric positive linear differential equations. Comm. Pure Appl. Math. XI (1958) 333-418.

[15] V. Gilrault and P.-A. Raviart, Finite element methods for the Navier-Stokes equatons, Theory and algorithms. Springer Ser. Comput. Math. 5 (1986).

[16] O.A. Karakashian and W.N. Jureidini, A nonconforming finite element method for the stationary Navier-Stokes equations. SIAM J. Numer. Anal. 35 (1998) 93-120.

[17] F. Li, C.-W. Shu, Locally divergence-free discontinuous Galerkin methods for MHD equations. SIAM J. Sci. Comput. 27 (2005) 413-442.

[18] J.-L. Lions and J. Petree, Sur une classe d'espaces d'interpolation. Publ. I.H.E.S. 19 (1964) 5-68.

[19] J.C. Nédélec, Mixed finite element in $\mathbb{R}^{3}$. Numer. Math. 35 (1980) 315-341. 
[20] K.G. Powell, An approximate Riemann solver for magnetohydrodynamics (that works in more than one dimension), ICASEReport 94-24 (NASA CR-194902), NASA Langley Research Center, Hampton, VA 23681-0001 (1994).

[21] P.-A. Raviart and J.M. Thomas, A mixed finite element method for 2nd order elliptic problems, Mathematical aspects of finite elements methods, in Proc. of the conference held in Rome, 10-12 Dec. 1975, A. Dold, B. Eckmann, Eds., Springer, Berlin, Heidelberg, New York. Lect. Notes Math. 606 (1977).

[22] G. Tóth, The $\nabla \cdot \mathbf{B}=0$ constraint in shock-capturing magnetohydrodynamics codes. J. Comput. Phys. 161 (2000) 605.

[23] L. Ying, A second order explicit finite element scheme to multidimensional conservation laws and its convergence. Sci. China Ser. A 43 (2000) 945-957.

[24] Q. Zhang and C.-W. Shu, Error Estimates to smooth solutions of Runge-Kutta discontinuous Galerkin methods for scalar conservation laws. SIAM J. Numer. Anal. 42 (2004) 641-666.

To access this journal online: www.edpsciences.org 